\title{
Partitioning gene-level contributions to complex-trait heritability by allele frequency identifies disease-relevant genes
}

Kathryn S. Burch*1†, Kangcheng Hou*1†, Yi Ding ${ }^{1}$, Yifei Wang ${ }^{2}$, Steven Gazal ${ }^{3}$, Huwenbo Shi ${ }^{4,5}$, and Bogdan Pasaniuc ${ }^{1,2,6,7, \dagger}$

1. Bioinformatics Interdepartmental Program, University of California, Los Angeles, Los Angeles, CA

2. Department of Pathology and Laboratory Medicine, David Geffen School of Medicine, University of California, Los Angeles, Los Angeles, CA

3. Center for Genetic Epidemiology, Department of Population and Public Health Sciences, Keck School of Medicine, University of Southern California, Los Angeles, CA

4. Department of Epidemiology, Harvard T.H. Chan School of Public Health, Boston, MA

5. Program in Medical and Population Genetics, Broad Institute of MIT and Harvard, Cambridge, MA

6. Department of Human Genetics, David Geffen School of Medicine, University of California, Los Angeles, Los Angeles, CA

7. Department of Computational Medicine, David Geffen School of Medicine, University of California, Los Angeles, CA

* These authors contributed equally to this work.

† Correspondence: K.S.B. (kathrynburch@ucla.edu), K.H. (kangchenghou@gmail.com), B.P. (pasaniuc@ucla.edu)

\section{Abstract}

Recent works have shown that SNP-heritability—which is dominated by low-effect common variants — may not be the most relevant quantity for localizing high-effect/critical disease genes. Here, we introduce methods to estimate the proportion of phenotypic variance explained by a given assignment of SNPs to a single gene (genelevel heritability). We partition gene-level heritability across minor allele frequency (MAF) classes to find genes whose gene-level heritability is explained exclusively by "low-frequency/rare" variants $(0.5 \% \leq \mathrm{MAF}<1 \%)$. Applying our method to $\sim 17 \mathrm{~K}$ protein-coding genes and 25 quantitative traits in the UK Biobank $(\mathrm{N}=290 \mathrm{~K})$, we find that, on average across traits, $\sim 2.5 \%$ of nonzero-heritability genes have a rare-variant component, and only $\sim 0.8 \%$ (370 gene-trait pairs) have heritability exclusively from rare variants. Of these 370 gene-trait pairs, 37\% were not detected by existing gene-level association testing methods, likely because existing methods combine signal from all variants in a region irrespective of MAF class. Many of the additional genes we identify are implicated in phenotypically related Mendelian disorders or congenital developmental disorders, providing further evidence of their trait-relevance. Notably, the rare-variant component of gene-level heritability exhibits trends different from those of common-variant gene-level heritability. For example, while total gene-level heritability increases with gene length, the rare-variant component is significantly larger among shorter genes; the cumulative distributions of gene-level heritability also vary across traits and reveal differences in the relative contributions of rare/common variants to overall gene-level polygenicity. We conclude that the proportion of gene-level heritability attributable to low-frequency/rare variation can yield novel insights into complex-trait genetic architecture. 


\section{Introduction}

Since the vast majority of risk variants identified through genome-wide association studies (GWAS) are located in noncoding regions, the genes and pathways driving complex traits are largely unknown ${ }^{1-3}$. For most complex traits, fundamental characteristics of genetic architecture-for example, the number of variants/genes with nonzero effects (polygenicity), the number of genes regulated by local versus distal variants, and the relative contributions of rare versus common variants to gene expression and phenotype-remain actively debated ${ }^{4-14}$.

That complex-trait SNP-heritability is enriched in regulatory regions is well established ${ }^{1,15-17}$. However, since SNP-heritability is overwhelmingly driven by common variants of low effect - individual rare variants with large per-allele effects contribute very little to population-level phenotypic variance ${ }^{18,19}$ — whether the largest heritability enrichments localize the most clinically relevant regions and/or genes for a trait is unclear. For example, a recent study estimates that the majority of complex-trait SNP-heritability mediated via the cis-genetic component of expression is explained by genes that individually have low cis-heritability of expression ${ }^{20}$. In addition, despite the inherent complexity of the biological processes driving complex traits, there is growing evidence that extreme complex-trait polygenicity may be explained in large part by negative/stabilizing selection, which purges high-effect alleles from the population, producing the remarkably even distribution of SNPheritability among common variants genome-wide (the so-called "flattening" hypothesis) $)^{21,22}$. If the most critical genes for a trait are not necessarily localized by enrichments of total heritability ${ }^{20,21,23,24}$, the open question of how to identify target genes using heritability enrichments or overlaps between GWAS and expression quantitative trait loci $^{25,26}$ becomes even murkier. Gene-based association tests that aggregate signal from multiple rare variants - for example, burden tests and sequence-based association tests (SKAT) — can increase power under different genetic-architecture scenarios ${ }^{27-36}$. However, such methods are generally designed to test for only rarevariant association or the combined effects of common and rare variants, and thus are not ideal for parsing the relative contributions of rare/common variants to the heritability of a single gene.

Here, we propose an approach to estimate the relative heritability contributions of common, low-frequency, and rare variants to a quantity we call gene-level heritability $\left(h_{\text {gene }}^{2}\right)$, defined as the proportion of phenotypic variance explained by the additive effects of a given set of variants assigned to a gene of interest. While the method itself is general and can be applied to any small annotation of interest (see Discussion), our goal in this work is to use MAF-partitioned gene-level heritability estimates to identify disease-relevant genes, which may have different relative contributions to heritability across MAF classes. The key challenge in estimating gene-level heritability lies in the uncertainty about which variants are causal and what their causal effect sizes are; such uncertainty in fine-mapping increases as the strength of LD in the region increases and as GWAS sample size decreases ${ }^{37}$. Consider a toy example in which a variant in the gene of interest is in perfect $L D(L D=1)$ with a second variant adjacent to the gene, the observed data are GWAS marginal association statistics and LD for the region (Figure 1a). Without additional information, it is impossible to definitively elucidate the underlying causal configuration. 
Even if the LD between the variants is 0.9 instead of 1, if this GWAS has $90 \%$ power to identify the associated region, to correctly reject the null hypothesis for the non-causal variant would require a sample size $\geq 4 \mathrm{x}$ larger than that of the original GWAS ${ }^{37}$. Since each causal configuration can yield a different gene-level heritability (with or without MAF-partitioning), randomly selecting one possible configuration (e.g., using variable selection methods such as the $\mathrm{Lasso}^{38}$ ) can yield inaccurate/misleading estimates. As an alternative approach, methods for partitioning genome-wide SNP-heritability across MAF bins can be employed. However, such methods are also ill-suited to our goals as they make distributional assumptions on the causal effects which (i) limit power to detect enrichment in small categories of variants $(<1 \%$ of the genome) and/or (ii) may not apply equally to rare and common variants ${ }^{15,17,39-43}$. Estimators for the SNP-heritability of a single region ("regional SNP-heritability") yield inflated estimates if any variants in the region of interest are in LD with the adjacent regions ${ }^{23,44-46}$. To address the fine-mapping uncertainty, we seek to propagate the uncertainty about which variants are causal to infer the posterior distribution over the entire gene of interest. Given GWAS summary statistics and estimates of $\mathrm{LD}$, we sample from the posterior distribution of the causal effect sizes within a probabilistic fine-mapping framework $^{47}$ and use the posterior samples to approximate the posterior distribution of gene-level heritability, thus capturing uncertainty in the causal effects (Figure 1b). From the full posterior distribution of gene-level heritability, one can compute various summary statistics of interest for each gene. We report the posterior mean, which we denote $\hat{h}_{\text {gene, }}^{2}$, and $\rho$-level credible intervals, or $\rho$-CI, defined as the central interval containing the true gene-level heritability with probability $\rho$ (Material and Methods).

We confirm in simulations that accounting for uncertainty in the estimated causal effects significantly reduces the

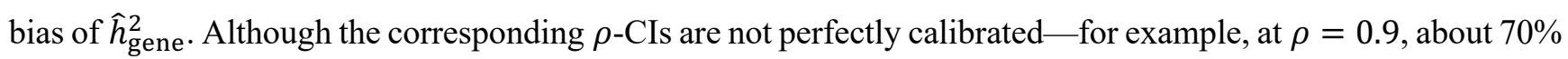
of credible intervals overlap $h_{\text {gene }}^{2}$ - among the true causal genes, any mis-calibrated CIs overwhelmingly tend to underestimate rather than overestimate $h_{\text {gene }}^{2}$. Both $\hat{h}_{\text {gene }}^{2}$ and $\rho$-CIs are robust to parameters such as causal effect sizes, gene length, allele frequencies of causal variants, and the strength of local LD. Assuming that total genelevel heritability can be expressed as $h_{\text {gene,t }}^{2}=h_{\text {gene,r }}^{2}+h_{\text {gene,lf }}^{2}+h_{\text {gene,c }}^{2}$, where each term refers to the component of $h_{\text {gene,t }}^{2}$ explained by rare $(0.5 \% \leq \mathrm{MAF}<1 \%)$, low-frequency $(1 \% \leq \mathrm{MAF}<5 \%)$, and common $(\mathrm{MAF} \geq 5 \%)$ variants, respectively, we apply the same approach to estimate the posterior distributions of $h_{\text {gene,r }}^{2}$, $h_{\text {gene,lf }}^{2}$, and $h_{\text {gene,c }}^{2}$ and observe similar trends and levels of accuracy (we note that there are many definitions of "rare" in the literature, and that we use $0.5 \% \leq \mathrm{MAF}<1 \%$ because we analyze imputed genotypes).

Applying our approach to estimate gene-level heritability for 17,436 genes and 25 quantitative traits in the UK Biobank $^{48}(\mathrm{~N}=290 \mathrm{~K}$ self-reported "white British", MAF $>0.5 \%)$, we find that $h_{\text {gene,t }}^{2}$ is indeed dominated by

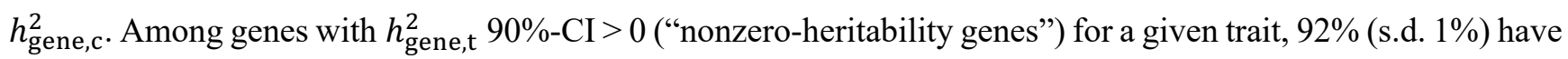
nonzero common-variant heritability, and $76 \%$ (s.d. 1\%) have nonzero heritability exclusively from common variants (i.e. $h_{\text {gene,t }}^{2} \approx h_{\text {gene,c }}^{2}$ ). In contrast, only $2.5 \%$ (s.d. $0.6 \%$ ) of nonzero-heritability genes, averaged across 
traits, have nonzero rare-variant heritability, and $0.8 \%$ (s.d. $0.4 \%$ ) have nonzero heritability exclusively from rare variants $\left(h_{\text {gene,t }}^{2} \approx h_{\text {gene,r }}^{2}\right)$. As a sanity check, we confirm that Mendelian-disorder genes from OMIM ${ }^{49}$, genes intolerant to loss of function (LoF) variants ${ }^{50}$, and a set of FDA-approved drug targets for 30 immune-related traits $^{51}$ have elevated estimates of all four heritability quantities (total, common, low-frequency, and rare). Among the $0.8 \%$ with $h_{\text {gene,t }}^{2} \approx h_{\text {gene,r }}^{2}(370$ gene-trait pairs in total), we identify many examples of disease genes with known roles in phenotypically similar Mendelian disorders and other congenital growth and developmental disorders. $37 \%$ of the 370 gene-trait pairs were not identified by existing methods for gene-level association testing, likely because existing methods have low power to detect genes containing only rare variants of moderate or low effect. We observe an overrepresentation of LoF-intolerant genes, but not Mendelian-disorder genes, among the $h_{\text {gene,t }}^{2} \approx h_{\text {gene,r }}^{2}$ genes. Using gene-level heritability estimates to further explore genetic architecture reveals notable differences between total/rare-variant gene-level heritability; for example, while total/common-variant gene-level heritability increases with gene length, we observe a clear inverse relationship between the rare-variant component and gene length.

Taken together, our results show that the low-frequency/rare-variant component of total gene-level heritability is useful for identifying narrow sets of high-impact genes that are not necessarily located in regions enriched with common-variant heritability. Our results are also consistent with the hypothesis that a sizable amount of complextrait variation is driven by dysregulation of genes that — if completely disrupted — cause phenotypically similar monogenic disorders and/or systemic congenital and developmental disorders ${ }^{52}$. Since some high-impact genes are disrupted/dysregulated by a combination of common and rare variants, we conclude that $h_{\text {gene,r }}^{2}$ should be considered alongside common-variant heritability enrichments if one is interested in identifying high-impact disease genes under different degrees of selection. While we restrict our analyses to genes $( \pm 10-\mathrm{kb}$ window), our method is general and can thus be applied to any small annotation of interest (e.g., enhancers, a set of genes involved in a pathway, a set of putative causal variants).

\section{Results}

\section{Overview of the Methods}

We propose a general approach for estimating the heritability explained by a given set of variants and assess its utility in estimating gene-level heritability. Given an assignment of $m$ variants to a gene $g$ of interest, total genelevel heritability is defined as $h_{\text {gene,t }}^{2} \equiv \operatorname{Var}\left[\mathbf{x}_{\mathrm{g}}^{T} \boldsymbol{\beta}_{\mathrm{g}} \mid \boldsymbol{\beta}\right]=\boldsymbol{\beta}_{\mathrm{g}}^{T} \mathbf{R}_{\mathrm{g}} \boldsymbol{\beta}_{\mathrm{g}}$, where $\boldsymbol{\beta}_{\mathrm{g}}$ is the $m \times 1$ vector of unknown causal effect sizes and $\mathbf{R}_{\mathrm{g}}$ is the $m \times m$ LD for SNPs in the gene (Material and Methods). Our goal in this work is to estimate a distribution over $h_{\text {gene, }}^{2}$ that captures uncertainty in the causal effects that arises from LD (Figure 1a). To this end, we adopt a probabilistic fine-mapping framework ${ }^{46,47}$ which assumes a sparse prior on the causal effect sizes in the LD block containing gene $g$ and infers the posterior distribution of the causal effect sizes, 
$p(\boldsymbol{\beta} \mid \widehat{\boldsymbol{\beta}}, \widehat{\mathbf{R}})$, where $\widehat{\boldsymbol{\beta}}$ is the vector of estimated marginal effects from GWAS and $\widehat{\mathbf{R}}$ is an estimate of LD. We sample from the posterior of $\boldsymbol{\beta}$ to approximate to the posterior of $h_{\text {gene,t }}^{2}$ (Figure 1b, Material and Methods). For each gene, we report the estimated posterior mean, denoted $\hat{h}_{\text {gene,t }}^{2}$, and $\rho$-level credible intervals ( $\rho$-CI), defined as the central interval that contains the true gene-level heritability with probability $\rho \in[0,1]$. Whereas previous works applied similar approaches to generate credible sets of causal variants ${ }^{47}$ or to estimate regional SNPheritability of LD blocks ${ }^{46}$, our goal in this work is to estimate the heritability explained by any arbitrary (not necessarily contiguous) set of variants much smaller than an LD block. This allows us to partition by minor allele frequency (MAF) bins under the assumption that $h_{\text {gene,t }}^{2}=h_{\text {gene,r }}^{2}+h_{\text {gene,lf }}^{2}+h_{\text {gene,c }}^{2}$, where the subscripts represent the rare $(0.5 \% \leq \mathrm{MAF}<1 \%)$, low-frequency $(1 \% \leq \mathrm{MAF}<5 \%)$, and common $(\mathrm{MAF} \geq 5 \%)$ variants assigned to the gene. (We note that, while there are many definitions of "rare" in the literature, we threshold at $\mathrm{MAF} \geq 0.5 \%$ because we want to reduce potential noise from imputation; see Discussion for details.)

\section{Accuracy of gene-level heritability estimates in simulations}

We perform simulations starting from real imputed genotypes of $\mathrm{N}=290,273$ "unrelated white British" individuals in the UK Biobank (chromosome 1, MAF > 0.5\%, M=200,235 variants, 1,083 genes; Material and Methods). In all simulations, the estimand of interest (gene-level heritability, $h_{\text {gene }}^{2}$ ) is the proportion of phenotypic variance explained by the variants in the gene body, as well as the MAF-partitioned counterpart. We note that our choice of variant assignment is arbitrary; there are many ways to assign variants to a gene, but our goal in this section is to provide a proof of concept. In brief, our simulation framework consists of three steps. First, for a given total heritability (variance explained by all $M$ variants) and cumulative gene-level heritability (variance explained by all genes), we randomly select $3 \%, 8 \%$, or $16 \%$ of the genes to be causal, where "causal" in this context refers to genes with $h_{\text {gene,t }}^{2}>0$. Second, for each causal gene, we draw causal variants in the gene body and within 10-kb upstream/downstream of the gene start/end positions; the purpose of the latter is to create situations where the estimated effects of variants in the region of interest are inflated in part because they tag causal effects located adjacent to the region. Third, we sample noncoding "background" causal variants from the whole chromosome with frequency $p_{\text {causal }}=\{0.001,0.01\}$. Under this model, the majority of simulated gene-level heritabilities are on the order of $10^{-6}$ to $10^{-3}$ (Supplementary Figure 1), similar to what we observe in real data in subsequent sections.

Overall, the estimated posterior means of total gene-level heritability, $\hat{h}_{\text {gene,t }}^{2}$, are highly concordant with the true gene-level heritabilities (Figure 2, Supplementary Figure 2). For each gene, we compute two metrics of accuracy from $s=30$ simulation replicates: $\operatorname{bias}\left[\hat{h}_{\text {gene, } \mathrm{t}}^{2}\right] \approx 1 / 30 \sum_{s}\left(\hat{h}_{\text {gene,t(s) }}^{2}-h_{\text {gene,t }}^{2}\right), \quad$ and $\operatorname{MSE}\left[\hat{h}_{\text {gene,t }}^{2}\right]=$ (bias $\left.\left[\hat{h}_{\text {gene,t }}^{2}\right]\right)^{2}+\operatorname{Var}\left[\hat{h}_{\text {gene,t }}^{2}\right]$ (mean squared error) (Material and Methods). As expected, MSE increases as the background polygenicity ( $\left.p_{\text {causal }}\right)$ and proportion of causal genes increase, i.e. as causal effect sizes of noncoding 
variants and gene-level heritabilities decrease (Supplementary Figure 3). Among the causal genes $\left(h_{\text {gene,t }}^{2}>0\right)$, $\hat{h}_{\text {gene,t }}^{2}$ tends to underestimate $h_{\text {gene,t }}^{2}$ with the median bias across genes ranging from approximately $-4 \% \times h_{\text {gene,t }}^{2}$ for lower polygenicities to $-30 \% \times h_{\text {gene,t }}^{2}$ for higher polygenicities (Figure 2, Supplementary Figure 4). There is a small positive correlation between bias and gene length (average Pearson $R=0.05$ (s.d. 0.02 ) across simulation setups), i.e. the estimates tend to be more downward-biased for shorter genes; average LD score and average MAF of variants in the gene have no discernible impact on accuracy (Supplementary Figures 5-8). To visualize the impact of causal-effect uncertainty on gene-level heritability estimation, we compare $\hat{h}_{\text {gene,t }}^{2}$ to a naive estimator that ignores LD between the gene and its adjacent regions, thus ignoring causal-effect uncertainty (Material and Methods). As expected, the naive estimator is significantly inflated; in particular, many noncausal genes have dramatically upward-biased estimates (Figure 2, Supplementary Figures 2 and 9) due to LD between variants in the gene and nearby causal variants. We benchmark the estimators for the contributions of rare, lowfrequency, and common variants to total gene-level heritability and find that they perform similarly to $\hat{h}_{\text {gene,t }}^{2}$ (Figure 3, Supplementary Figures 3, 4, 6-8, 10-12).

\section{Calibration of credible intervals}

Calibration of $\rho$-level credible intervals ( $\rho$-CIs) was assessed using "empirical coverage," defined here as the proportion of simulation replicates in which $\rho$-CI contains the true gene-level heritability (Material and Methods). Perfect calibration of $\rho$-CI would manifest as empirical coverage equal to $\rho$ for all $\rho \in[0,1]$. In reality, we observe a downward bias in empirical coverage across all simulations that increases in magnitude as the proportion of causal genes increases (i.e. as per-variant causal effect sizes decrease). For example, at $\rho=0.9$, empirical coverage ranges from approximately 0.75 when $3 \%$ of genes are causal to 0.65 when $16 \%$ are causal (Supplementary Figure 13). While downward bias in empirical coverage can be the result of $\rho$-CIs underestimating or overestimating $h_{\text {gene,t }}^{2}$, the credible intervals at $\rho=\{0.90,0.95\}$ tend to underestimate the true gene-level heritability (Supplementary Table 1), consistent with the downward-bias we observe in $\hat{h}_{\text {gene,t }}^{2}$ (Figure 2). For example, at $\rho=0.95$, the proportion of true causal genes that are underestimated vs. overestimated is approximately $14 \%$ vs. $6 \%$ (when $3 \%$ of genes are causal) and $30 \%$ vs. $3.5 \%$ (when $16 \%$ of genes are causal) (Supplementary Table 1). The $\rho$-CIs for $h_{\text {gene,r }}^{2}$ are more conservative; for the same parameters, among the genes with true $h_{\text {gene,r }}^{2}>0$, the proportions of underestimated vs overestimated genes are $38 \%$ vs. $1.5 \%$ (when $3 \%$ of genes are causal) and $45 \%$ vs. $<1 \%$ (when $16 \%$ of genes are causal) (Supplementary Table 2, Supplementary Figure 14).

\section{Robustness to noise in estimates of $\mathrm{LD}$}

Finally, we assess whether $\hat{h}_{\text {gene,t }}^{2}$ is robust to the number of individuals used to estimate LD, i.e. the sample size of the "LD panel" (Material and Methods). Compared to in-sample LD computed from the full set of individuals 
in the GWAS $(\mathrm{N}=290,273)$, using a random subset of $\mathrm{N}=\{500,1000,2500,5000\}$ individuals from the original GWAS does not significantly impact the MSE of $\hat{h}_{\text {gene,t }}^{2}$ or $\hat{h}_{\text {gene,r }}^{2}$ (Supplementary Figure 15 ). Using 90\%-CIs to identify potential causal genes (i.e. 90\%-CI lower bound >0), we observe a slight increase in the false positive rate for both $\hat{h}_{\text {gene,t }}^{2}$ and $\hat{h}_{\text {gene,r }}^{2}$ as $\mathrm{N}$ decreases (Supplementary Figure 16); this is accompanied by a slight increase in power for $\hat{h}_{\text {gene,t }}^{2}$ but not for $\hat{h}_{\text {gene,r }}^{2}$ (Supplementary Figure 17). Since the N=5,000 LD panel and the full in-sample LD yield similar false positive rates for both estimators, we recommend using an in-sample LD panel of no less than 5,000 individuals (see Discussion for additional comments on LD panels).

\section{$h_{\text {gene, }}^{2}$ identifies genes that link complex traits to phenotypically related monogenic disorders}

We estimate, and partition by MAF, the gene-level heritabilities of 17,437 genes for 25 quantitative traits in the UK Biobank ( $\mathrm{N}=290,273$ "unrelated white British" individuals ${ }^{48}, \mathrm{M}=5,650,812$ with $\mathrm{MAF}>0.5 \%$, imputed data; Material and Methods). Unless otherwise stated, the quantity of interest, $h_{\text {gene,t }}^{2}$, is a function of the variants located in the gene body and the variants located within 10-kb upstream/downstream from the gene start/end positions. A gene is classified as having "nonzero heritability" if it meets two criteria: (i) the 90\%-CI for $h_{\text {gene,t }}^{2}$ does not overlap zero and (ii) the $90 \%$-CI for at least one MAF component $\left(h_{\text {gene,r }}^{2}, h_{\text {gene,lf }}^{2}\right.$, or $\left.h_{\text {gene,c }}^{2}\right)$ does not overlap zero. Using this definition, the number of nonzero-heritability genes ranges from 1,212 (7\%) for corneal hysteresis to 2,469 (14\%) for height (Table 1). Most of the estimated posterior means for these genes lie between $10^{-6}$ and $10^{-4}$ (Figure 4).

As expected, $\hat{h}_{\text {gene,c }}^{2}$ behaves similarly to $\hat{h}_{\text {gene,t }}^{2}$ The average Pearson $\mathrm{R}^{2}$ of $\hat{h}_{\text {gene,c }}^{2}$ and $\hat{h}_{\text {gene,t }}^{2}$ across the 25 traits is $94 \%$ (s.d. 1\%) (Figure 4, Supplementary Figure 18). 92\% (s.d. 1\%) of nonzero-heritability genes have significant common-variant heritability; $76 \%$ (s.d. $1 \%$ ) have significant causal effects exclusively from common variants (Table 1). On the other hand, $\hat{h}_{\text {gene, } r}^{2}$ is significantly less correlated with $\hat{h}_{\text {gene,t }}^{2}$ (average $\mathrm{R}^{2}=30 \%$ (s.d. 21\%) across traits) (Figure 4, Supplementary Figure 18). Approximately 2.5\% (s.d. 0.6\%) of genes have significant rare-variant heritability, and only $0.8 \%$ (s.d. $0.4 \%$ ) - 370 gene-trait pairs in total—have significant heritability exclusively from rare variants (Table 1, Supplementary Table 3). Of these 370 gene-trait pairs with only rare-variant heritability (ranging from 4 genes for heel T-score and corneal hysteresis to 32 genes for height (Table 1, Supplementary Table 3)), 232 gene-trait pairs are also identified by MAGMA ${ }^{53}$ (FDR $<0.05$, Material and Methods). These 232 gene-trait pairs have a median $\hat{h}_{\text {gene,t }}^{2} \approx \hat{h}_{\text {gene,r }}^{2}$ on the order of $10^{-4}$ whereas the median for the remaining gene-trait pairs not found by MAGMA is $\sim 10^{-6}$. This suggests that MAGMA likely has limited power to detect signal from rare causal variants of moderate effect, which is expected as MAGMA tests for association between the total causal-variant signal at a gene and phenotype; it is not designed for partitioning the signal into components from different allele-frequency classes. 
The 138 additional gene-trait pairs identified with our approach (Supplementary Table 4) include several genes implicated in phenotypically related Mendelian disorders. For example, AKT2 is identified for serum gammaglutamyl transferase $\left(90 \%-\mathrm{CI}\right.$ of $h_{\text {gene,t }}^{2}=\left[3 \times 10^{-5}, 1 \times 10^{-4}\right]$, MAGMA z-score: 1.1$)$, which is used to test for the presence of liver disease; AKT2 is implicated in monogenic forms of type 2 diabetes $^{54}$ and hypoinsulinemic hypoglycemia with hemihypertrophy ${ }^{55}$. The $A K T 2$ annotation used for this analysis contains a total of 104 variants; 24 are rare variants, of which 1 is identified as causal. For serum alkaline phosphatase (used to diagnose diseases related to the liver or skeletal system), we identify MDM4 (90\%-CI of $h_{\text {gene,t }}^{2}=\left[4 \times 10^{-7}, 5 \times 10^{-6}\right]$, MAGMA z-score: 1.3; annotation contains 273 variants; 144 are rare variants, of which $\sim 5$ are identified as causal), which encodes a negative regulator of p53-mediated transcription ${ }^{56}$ that was recently implicated in an autosomal dominant bone marrow failure syndrome ${ }^{57}$. COL4A4, identified for serum apolipoprotein A1 (a test for atherosclerotic cardiovascular disease; $90 \%$-CI of $h_{\text {gene,t }}^{2}=\left[4 \times 10^{-5}, 2 \times 10^{-4}\right]$; MAGMA z-score: 1.1; annotation contains 390 variants; 33 are rare variants, of which $\sim 1$ is identified as causal), is implicated in monogenic forms of kidney disease ranging in severity from hematuria to end-stage renal disease $\mathrm{e}^{58-61}$.

We also identify several genes implicated in congenital developmental and metabolic disorders. For example, RTTN, identified for mean corpuscular hemoglobin $\left(90 \%-\mathrm{CI}\right.$ of $h_{\text {gene,t }}^{2}=\left[9 \times 10^{-6}, 2 \times 10^{-4}\right]$; MAGMA zscore: 2.2; annotation contains 369 variants; 83 are rare, of which 2 are identified as causal), is implicated in microcephaly, short stature, and polymicrogyria with seizures ${ }^{62-65}$. SLC25A24, identified for serum cystatin C $\left(90 \%\right.$-CI of $h_{\text {gene,t }}^{2}=\left[3 \times 10^{-5}, 2 \times 10^{-4}\right]$; MAGMA z-score: 1.8; annotation contains 243 variants; 21 are rare, of which $\sim 1$ is causal), is implicated in Fontaine progeroid syndrome ${ }^{66,67} . T B C K$, identified for red blood cell count $\left(90 \%\right.$-CI of $h_{\text {gene,t }}^{2}=\left[3 \times 10^{-5}, 2 \times 10^{-4}\right]$; MAGMA z-score: 2.0 ; annotation contains 617 variants; 59 are rare, of which $\sim 1$ is causal), is implicated in infantile hypotonia with psychomotor retardation and characteristic facies $^{68-70}$.

Taken together, these findings indicate that the rare-variant contribution to total gene-level heritability is indeed useful for identifying disease-relevant genes, especially those with moderate or relatively low total heritability, which existing methods can be underpowered to detect. Our results are consistent with the hypothesis that complex-trait variation may be explained in part by dysregulation of genes that —if completely disrupted — cause phenotypically similar or related Mendelian disorders ${ }^{52}$. We emphasize that, since heritability reflects genetic and phenotypic variation at the population level, if a common variant and rare variant explain the same heritability (i.e. have the same standardized causal effect size), the allelic effect - the expected change in phenotype per additional copy of the effect allele - is significantly larger for the rare variant. 


\section{LoF-intolerant genes are overrepresented among genes with only rare-variant heritability}

We estimate, and partition by MAF, the gene-level heritabilities of three gene sets: (i) known Mendelian-disorder genes from $\operatorname{OMIM}^{49}(\mathrm{n}=3,446)$, (ii) loss-of-function $(\mathrm{LoF})$-intolerant genes (probability of LoF-intolerance $(\mathrm{pLI})>$ $0.9)^{50}(\mathrm{n}=3,230)$, and (iii) a set of FDA-approved drug targets for 30 immune-related traits ${ }^{51}(\mathrm{n}=216)$ (Material and Methods). Compared to a set of "null" genes (sampled from the set of genes not contained in any of the three gene sets), all three gene sets have significantly higher median estimates of total and MAF-partitioned gene-level heritability (Figure 5).

We investigate whether certain classes of nonzero-heritability genes are overrepresented in the Mendeliandisorder and LoF-intolerant gene sets. The Mendelian-disorder gene set comprises $\sim 20 \%$ of all genes and is enriched for genes with nonzero heritability for at least one trait (Fisher's exact test, 95\%-CI of OR: [1.2, 1.4]); the number of genes in both categories ranges from 261 for corneal hysteresis to 557 for height. The LoF-intolerant genes comprise $\sim 19 \%$ of all genes and are also enriched for nonzero-heritability genes (Fisher's exact test, $95 \%$ CI of OR: [1.5, 1.7]); the overlap between the two categories ranges from 314 genes for corneal hysteresis to 650 for height. In contrast, genes with exclusively rare-variant heritability are significantly enriched in the LoFintolerant gene set (95\%-CI of OR: $[1.1,2.1])$ but not in the Mendelian-disorder gene set (95\% CI of OR: [0.9, 1.7]). On average across traits, $\sim 19 \%$ (s.d. $11 \%$ ) of the previously identified $h_{\text {gene,t }}^{2}=h_{\text {gene, }}^{2}$ genes and $\sim 21 \%$ (s.d. 1\%) of genes with only common-variant heritability are also in the Mendelian-disorder gene set. In contrast, $\sim 32 \%$ (s.d. $16 \%$ ) of genes with $h_{\text {gene,t }}^{2}=h_{\text {gene,r }}^{2}$ are also in the LoF-intolerant gene set, compared with $\sim 23 \%$ (s.d. $1 \%$ ) of genes with $h_{\text {gene,t }}^{2}=h_{\text {gene,c }}^{2}$.

\section{MAF-partitioned gene-level heritability reveals unique insights into genetic architecture}

We investigated whether gene-level heritability estimates are correlated with gene length, average LD score of variants in the gene (a proxy for the strength of LD in the region), and average MAF of variants in the gene. $h_{\text {gene,c }}^{2}$ (and, to a large extent, $h_{\text {gene,lf }}^{2}$ ) is distributed very similarly to $h_{\text {gene,t }}^{2}$ with respect to these variables (Figure 6, Supplementary Figure 19). However, the distribution of $h_{\text {gene,r }}^{2}$ shows marked differences, particularly with respect to gene length. Specifically, we observe higher average $h_{\text {gene,r }}^{2}$ among shorter genes even though the number of causal variants per gene (across all allele frequencies) increases with gene length (Figure 6, Supplementary Figure 20). The expected per-causal variant effect size per gene is invariant to gene length for common and low-frequency variants, but for rare variants, the average across gene-trait pairs is nearly $10^{-4}$ in the shortest quintile of genes versus $10^{-6}$ in the longest (Figure 6). While this result initially seems paradoxical, it is not inconsistent with the literature; previous studies have reported strong inverse correlations between gene length and expression which could be due to, for example, natural selection favoring fewer/shorter introns in highly expressed genes due to the high energy/costs associated with transcription and splicing ${ }^{71,72}$. 
Using the empirical distributions of cumulative $h_{\text {gene,t }}^{2}, h_{\text {gene,c }}^{2}, h_{\text {gene,lf }}^{2}$, and $h_{\text {gene,r }}^{2}$, we loosely quantify differences in polygenicity at the level of genes (with the caveat that, since there is a high degree of gene overlap in some regions, cumulative $h_{\text {gene,t }}^{2}$ may be more informative for some traits over others) (Figure 7). For example, if cumulative $h_{\text {gene,t }}^{2}$ is divided equally among nonzero-heritability genes, the empirical CDF for $h_{\text {gene,t }}^{2}$ would be the line $y=x$, where the $\mathrm{x}$-axis is the rank ordering of genes from highest to lowest $h_{\text {gene,t }}^{2}$; two traits with the same empirical CDF for $h_{\text {gene,t }}^{2}$ can have different empirical CDFs for each MAF-partitioned component. Once again, we find that the cumulative distributions of $h_{\text {gene,c }}^{2}$ are extremely similar to those of $h_{\text {gene,t }}^{2}$ (Figure 7, Supplementary Figure 21). Although the curves generally have similar shapes across traits (i.e. similar spread of heritability across genes), some traits have a notable amount of heritability concentrated in just the top gene, and many of these gene-trait pairs have been functionally validated in the literature. For example, for serum urate concentration, $S L C 2 A 9$ - a known urate transporter ${ }^{73-75}$ - is the single largest contributor to total, common-, and LF-variant gene-level heritability $\left(\hat{h}_{\text {gene,t }}^{2}=6.2 \%, \hat{h}_{\text {gene,c }}^{2}=5.9 \%, \hat{h}_{\text {gene,lf }}^{2}=0.3 \%, \hat{h}_{\text {gene,r }}^{2}=0\right)$, accounting for $46 \%, 51 \%$, and $29 \%$ of the cumulative heritability for each estimand, respectively (Figure 7); certain loss-of-function mutations in $S L C 2 A 9$ are known to cause a rare form of renal hypouricemia ${ }^{76-78}$, a disorder characterized in part by low serum urate levels. For serum alkaline phosphatase, we find that $A L P L-$ which encodes the enzyme alkaline phosphatase - is the single largest contributor to total and LF-variant gene-level heritability $\left(\hat{h}_{\text {gene,t }}^{2}=4.1 \%, \hat{h}_{\text {gene,c }}^{2}=1.8 \%, \hat{h}_{\text {gene,lf }}^{2}=2.1 \%, \hat{h}_{\text {gene,r }}^{2}=0 \%\right)$, explaining $15 \%$ and $39 \%$ of the respective cumulative heritability estimands (Figure 7); certain loss-of-function mutations in $A L P L$ are known to cause hypophosphatasia, a monogenic disorder characterized in part by low alkaline phosphatase ${ }^{79,80}$.

\section{Discussion}

We propose a general approach for estimating the heritability explained by any set of variants much smaller than an LD block and assess its utility in estimating/partitioning gene-level heritability. In simulations, we confirm that incorporating uncertainty about which variants are causal and what their effect sizes are dramatically improves specificity over naive approaches that ignore uncertainty in the causal effects. For 25 complex traits and $>17 \mathrm{~K}$ genes, we estimate gene-level heritability - the heritability explained by variants in the gene body plus a 10-kb window upstream/downstream from the gene start/end positions - and partition by allele-frequency class to explore differences in genetic architecture across traits. As expected, most gene-level heritability is dominated by common variants, but we identify several genes with nonzero heritability exclusively from rare or low-frequency variants. Notably, we identify many genes with nonzero gene-level heritability explained exclusively by rare variants that existing methods are underpowered to detect. Many of these genes have known roles in Mendelian disorders that are phenotypically similar or related to the complex trait; we also identify genes implicated in systemic congenital developmental and metabolic disorders. Our results demonstrate that the rare-variant 
contribution to total gene-level heritability is a useful quantity that can be considered alongside common-variant heritability enrichments to obtain a more comprehensive understanding of genetic architecture.

We conclude by discussing the limitations of our approach. First, multiple lines of evidence suggest that rare and "ultra-rare" variants, which are not well-tagged by variants on genotyping arrays, may explain much of the "missing heritability" not captured by genotyped or imputed variants ${ }^{81-84}$. Since imputed genotypes are noisier for rarer variants and variants in lower LD regions, we analyze variants with MAF $>0.5 \%$. Additional work is needed to assess the error incurred by using genotyped/imputed data in lieu of whole genome sequencing (WGS) as well as the signal that is missed by excluding variants with $\mathrm{MAF}<0.5 \%$. While our estimator can be applied to whole exome sequencing (WES) data, LD between coding and noncoding regions would significantly inflate gene-level heritability estimates; LD between exonic and intronic variants could also cloud interpretation, depending on the application. With multiple biobanks starting to sequence large numbers of individuals ${ }^{85-88}$, we believe the availability of large-scale WGS data will gradually become less of an issue.

We correct for population structure using genome-wide principal components (PCs) computed from the same imputed genotypes that are used to perform each GWAS. This is a standard approach to correcting for population stratification, which typically reflects geographic separation, in estimates of genome-wide SNP-heritability and genome-wide functional enrichments, both of which are driven by common SNPs. However, rare variants generally have more complex spatial distributions and thus exhibit stratification patterns distinct from those of common SNPs ${ }^{84,89}$. It is unclear whether methods that are effective for controlling stratification of common SNPs are applicable to rare variants ${ }^{90}$. We leave the question of whether uncorrected structure among rare variants significantly influences our estimates of gene-level heritability for future work.

Our approach requires OLS association statistics and LD computed from a subset of individuals in the GWAS. While estimates of gene-level heritability and the MAF-partitioned components are robust to sample sizes as low as 5,000, the individuals used to estimate LD must be a subset of the individuals in the GWAS. Although summary association statistics are publicly available for hundreds of large-scale GWAS, most of these studies are metaanalyses and therefore do not have in-sample LD available. Moreover, many publicly available summary statistics were computed from linear mixed models rather than OLS, which is used throughout our simulations and derivations. Additional work is needed to extend our approach to allow external reference panel LD (e.g., 1000 Genomes $^{91}$ ) and/or mixed model association statistics. Biobanks can help to ameliorate potential issues stemming from noisy LD by releasing summary LD information in addition to summary association statistics ${ }^{92}$.

Finally, gene-level heritabilities of different genes can have nonzero covariance due to physical overlap between genes and/or correlated causal effect sizes. Thus, the heritability estimates reported in this work have additional sources of noise/uncertainty which were not directly modeled or accounted for. Since modeling correlation of 
causal effect sizes would make inference considerably more challenging, we leave this for future work.

Importantly, genes with credible intervals $>0$ should not be interpreted as "causal" for the complex trait without additional functional validation, as nonzero gene-level heritability indicates association but not causality.

\section{Declaration of Interests}

The authors declare no competing interests.

\section{Acknowledgments}

We thank the UK Biobank Resource (application \#33297) for making this work possible. We are also grateful to Alkes Price, Harold Pimentel, Luke O’Connor, Nasa Sinnott-Armstrong, and Ruth Johnson for providing helpful comments and discussion. This work was funded in part by the National Institutes of Health under awards R01HG009120, R01-MH115676.

\section{Web Resources}

Protein-coding gene list: https://github.com/bogdanlab/gene sets/protein coding genes.bed

OMIM gene list: https://github.com/bogdanlab/gene sets/blob/master/mendelian_genes.bed

LoF-intolerance metrics by gene: https://gnomad.broadinstitute.org/downloads

susieR software: https://github.com/stephenslab/susieR

MAGMA software: https://ctg.cncr.nl/software/magma

PLINK software: https://www.cog-genomics.org/plink2

The UK Biobank Resource: https://www.ukbiobank.ac.uk/ 


\section{References}

1. Maurano, M. T. et al. Systematic localization of common disease-associated variation in regulatory DNA. Science 337, 1190-1195 (2012).

2. Visscher, P. M. et al. 10 years of GWAS discovery: Biology, function, and translation. Am. J. Hum. Genet. 101, 5-22 (2017).

3. Cano-Gamez, E. \& Trynka, G. From GWAS to function: Using functional genomics to identify the mechanisms underlying complex diseases. Front. Genet. 11, 424 (2020).

4. Wray, N. R., Wijmenga, C., Sullivan, P. F., Yang, J. \& Visscher, P. M. Common disease is more complex than implied by the core gene omnigenic model. Cell 173, 1573-1580 (2018).

5. Boyle, E. A., Li, Y. I. \& Pritchard, J. K. An expanded view of complex traits: From polygenic to omnigenic. Cell 169, 1177-1186 (2017).

6. Liu, X., Li, Y. I. \& Pritchard, J. K. Trans effects on gene expression can drive omnigenic inheritance. Cell 177, $1022-$ 1034.e6 (2019).

7. Bomba, L., Walter, K. \& Soranzo, N. The impact of rare and low-frequency genetic variants in common disease. Genome Biol. 18, (2017).

8. Yao, C. et al. Dynamic role of trans regulation of gene expression in relation to complex traits. Am. J. Hum. Genet. 100, 985-986 (2017).

9. Pritchard, J. K. Are rare variants responsible for susceptibility to complex diseases? Am. J. Hum. Genet. 69, 124-137 (2001).

10. Caballero, A., Tenesa, A. \& Keightley, P. D. The nature of genetic variation for complex traits revealed by GWAS and regional heritability mapping analyses. Genetics 201, 1601-1613 (2015).

11. Golan, D., Lander, E. S. \& Rosset, S. Measuring missing heritability: inferring the contribution of common variants. Proc. Natl. Acad. Sci. U. S. A. 111, E5272-81 (2014).

12. Eyre-Walker, A. Evolution in health and medicine Sackler colloquium: Genetic architecture of a complex trait and its implications for fitness and genome-wide association studies. Proc. Natl. Acad. Sci. U. S. A. 107 Suppl 1, 1752-1756 (2010).

13. Pritchard, J. K. \& Cox, N. J. The allelic architecture of human disease genes: common disease-common variant...or not? Hum. Mol. Genet. 11, 2417-2423 (2002).

14. Wainschtein, P. et al. Recovery of trait heritability from whole genome sequence data. bioRxiv (2019) doi:10.1101/588020.

15. Finucane, H. K. et al. Partitioning heritability by functional annotation using genome-wide association summary statistics. Nat. Genet. 47, 1228-1235 (2015).

16. Pickrell, J. K. Joint analysis of functional genomic data and genome-wide association studies of 18 human traits. $\mathrm{Am}$. J. Hum. Genet. 95, 126 (2014).

17. Gusev, A. et al. Partitioning heritability of regulatory and cell-type-specific variants across 11 common diseases. $A m$. J. Hum. Genet. 95, 535-552 (2014).

18. Yang, J. et al. Common SNPs explain a large proportion of the heritability for human height. Nat. Genet. 42, 565-569 (2010).

19. Hunt, K. A. et al. Negligible impact of rare autoimmune-locus coding-region variants on missing heritability. Nature 498, 232-235 (2013).

20. Yao, D. W., O'Connor, L. J., Price, A. L. \& Gusev, A. Quantifying genetic effects on disease mediated by assayed gene expression levels. Nat. Genet. 52, 626-633 (2020).

21. O'Connor, L. J. et al. Extreme polygenicity of complex traits is explained by negative selection. Am. J. Hum. Genet. 105, 456-476 (2019).

22. Simons, Y. B., Bullaughey, K., Hudson, R. R. \& Sella, G. A population genetic interpretation of GWAS findings for human quantitative traits. PLoS Biol. 16, e2002985 (2018).

23. Gusev, A. et al. Quantifying missing heritability at known GWAS loci. PLoS Genet. 9, e1003993 (2013).

24. Marouli, E. et al. Rare and low-frequency coding variants alter human adult height. Nature 542, 186-190 (2017).

25. Gusev, A. et al. Integrative approaches for large-scale transcriptome-wide association studies. Nat. Genet. 48, 245 252 (2016). 
26. Wainberg, M. et al. Opportunities and challenges for transcriptome-wide association studies. Nat. Genet. 51, 592-599 (2019).

27. Ionita-Laza, I., Lee, S., Makarov, V., Buxbaum, J. D. \& Lin, X. Sequence kernel association tests for the combined effect of rare and common variants. Am. J. Hum. Genet. 92, 841-853 (2013).

28. Wu, M. C. et al. Rare-variant association testing for sequencing data with the sequence kernel association test. Am. J. Hum. Genet. 89, 82-93 (2011).

29. Price, A. L. et al. Pooled association tests for rare variants in exon-resequencing studies. Am. J. Hum. Genet. 86, 832838 (2010).

30. Zuk, O. et al. Searching for missing heritability: designing rare variant association studies. Proc. Natl. Acad. Sci. U. S. A. 111, E455-64 (2014).

31. Zhan, X., Hu, Y., Li, B., Abecasis, G. R. \& Liu, D. J. RVTESTS: an efficient and comprehensive tool for rare variant association analysis using sequence data. Bioinformatics 32, 1423-1426 (2016).

32. Moutsianas, L. et al. The power of gene-based rare variant methods to detect disease-associated variation and test hypotheses about complex disease. PLoS Genet. 11, e1005165 (2015).

33. Liu, D. J. et al. Meta-analysis of gene-level tests for rare variant association. Nat. Genet. 46, 200-204 (2014).

34. Lee, S., Abecasis, G. R., Boehnke, M. \& Lin, X. Rare-variant association analysis: study designs and statistical tests. Am. J. Hum. Genet. 95, 5-23 (2014).

35. Lee, S. et al. Optimal unified approach for rare-variant association testing with application to small-sample casecontrol whole-exome sequencing studies. Am. J. Hum. Genet. 91, 224-237 (2012).

36. Lee, S., Wu, M. C. \& Lin, X. Optimal tests for rare variant effects in sequencing association studies. Biostatistics 13, $762-775$ (2012).

37. Udler, M. S., Tyrer, J. \& Easton, D. F. Evaluating the power to discriminate between highly correlated SNPs in genetic association studies. Genet. Epidemiol. 34, 463-468 (2010).

38. Tibshirani, R. Regression shrinkage and selection via the lasso. J. R. Stat. Soc. 58, 267-288 (1996).

39. Loh, P.-R. et al. Contrasting genetic architectures of schizophrenia and other complex diseases using fast variancecomponents analysis. Nat. Genet. 47, 1385-1392 (2015).

40. Gazal, S. et al. Functional architecture of low-frequency variants highlights strength of negative selection across coding and non-coding annotations. Nat. Genet. 50, 1600-1607 (2018).

41. Pazokitoroudi, A. et al. Efficient variance components analysis across millions of genomes. Nat. Commun. 11, 4020 (2020).

42. Speed, D. \& Balding, D. J. SumHer better estimates the SNP heritability of complex traits from summary statistics. Nat. Genet. 51, 277-284 (2019).

43. Yang, J. et al. Genetic variance estimation with imputed variants finds negligible missing heritability for human height and body mass index. Nat. Genet. 47, 1114-1120 (2015).

44. Gamazon, E. R., Cox, N. J. \& Davis, L. K. Structural architecture of SNP effects on complex traits. Am. J. Hum. Genet. 95, 477-489 (2014).

45. Shi, H., Kichaev, G. \& Pasaniuc, B. Contrasting the genetic architecture of 30 complex traits from summary association data. Am. J. Hum. Genet. 99, 139-153 (2016).

46. Benner, C., Havulinna, A. S., Salomaa, V., Ripatti, S. \& Pirinen, M. Refining fine-mapping: effect sizes and regional heritability. bioRxiv (2018) doi:10.1101/318618.

47. Wang, G., Sarkar, A., Carbonetto, P. \& Stephens, M. A simple new approach to variable selection in regression, with application to genetic fine mapping. J. R. Stat. Soc. Series B Stat. Methodol. 82, 1273-1300 (2020).

48. Bycroft, C. et al. The UK Biobank resource with deep phenotyping and genomic data. Nature 562, 203-209 (2018).

49. Amberger, J. S., Bocchini, C. A., Schiettecatte, F., Scott, A. F. \& Hamosh, A. OMIM.org: Online Mendelian Inheritance in Man (OMIM $\left.{ }^{\circledR}\right)$, an online catalog of human genes and genetic disorders. Nucleic Acids Res. 43, D78998 (2015).

50. Lek, M. et al. Analysis of protein-coding genetic variation in 60,706 humans. Nature 536, 285-291 (2016).

51. Fang, H. et al. A genetics-led approach defines the drug target landscape of 30 immune-related traits. Nat. Genet. 51, 1082-1091 (2019).

52. Freund, M. K. et al. Phenotype-specific enrichment of Mendelian disorder genes near GWAS regions across 62 complex traits. Am. J. Hum. Genet. 103, 535-552 (2018). 
53. de Leeuw, C. A., Mooij, J. M., Heskes, T. \& Posthuma, D. MAGMA: generalized gene-set analysis of GWAS data. PLoS Comput. Biol. 11, e1004219 (2015).

54. George, S. et al. A family with severe insulin resistance and diabetes due to a mutation in AKT2. Science 304, 13251328 (2004).

55. Hussain, K. et al. An activating mutation of AKT2 and human hypoglycemia. Science 334, 474-474 (2011).

56. Biderman, L., Manley, J. L. \& Prives, C. Mdm2 and MdmX as regulators of gene expression. Genes Cancer 3, 264273 (2012).

57. Toufektchan, E. et al. Germline mutation of MDM4, a major p53 regulator, in a familial syndrome of defective telomere maintenance. Sci. Adv. 6, eaay3511 (2020).

58. Mencarelli, M. A. et al. Evidence of digenic inheritance in Alport syndrome. J. Med. Genet. 52, 163-174 (2015).

59. Mochizuki, T. et al. Identification of mutations in the alpha 3(IV) and alpha 4(IV) collagen genes in autosomal recessive Alport syndrome. Nat. Genet. 8, 77-81 (1994).

60. Lemmink, H. H. et al. Benign familial hematuria due to mutation of the type IV collagen alpha4 gene. J. Clin. Invest. 98, 1114-1118 (1996).

61. Badenas, C. et al. Mutations in theCOL4A4 and COL4A3 genes cause familial benign hematuria. J. Am. Soc. Nephrol. 13, 1248-1254 (2002).

62. Kheradmand Kia, S. et al. RTTN mutations link primary cilia function to organization of the human cerebral cortex. Am. J. Hum. Genet. 91, 533-540 (2012).

63. Shamseldin, H. et al. RTTN mutations cause primary microcephaly and primordial dwarfism in humans. Am. J. Hum. Genet. 97, 862-868 (2015).

64. Rump, P. et al. Whole-exome sequencing is a powerful approach for establishing the etiological diagnosis in patients with intellectual disability and microcephaly. BMC Med. Genomics 9, 7 (2016).

65. Shaheen, R. et al. Genomic and phenotypic delineation of congenital microcephaly. Genet. Med. 21, 545-552 (2019).

66. Writzl, K. et al. De Novo mutations in SLC25A24 cause a disorder characterized by early aging, bone dysplasia, characteristic face, and early demise. Am. J. Hum. Genet. 101, 844-855 (2017).

67. Ehmke, N. et al. De Novo Mutations in SLC25A24 Cause a Craniosynostosis Syndrome with Hypertrichosis, Progeroid Appearance, and Mitochondrial Dysfunction. Am. J. Hum. Genet. 101, 833-843 (2017).

68. Alazami, A. M. et al. Accelerating novel candidate gene discovery in neurogenetic disorders via whole-exome sequencing of prescreened multiplex consanguineous families. Cell Rep. 10, 148-161 (2015).

69. Chong, J. X. et al. Recessive inactivating mutations in TBCK, encoding a Rab GTPase-activating protein, cause severe infantile syndromic encephalopathy. Am. J. Hum. Genet. 98, 772-781 (2016).

70. Bhoj, E. J. et al. Mutations in TBCK, encoding TBC1-domain-containing kinase, lead to a recognizable syndrome of intellectual disability and hypotonia. Am. J. Hum. Genet. 98, 782-788 (2016).

71. Castillo-Davis, C. I., Mekhedov, S. L., Hartl, D. L., Koonin, E. V. \& Kondrashov, F. A. Selection for short introns in highly expressed genes. Nat. Genet. 31, 415-418 (2002).

72. Grishkevich, V. \& Yanai, I. Gene length and expression level shape genomic novelties. Genome Res. 24, 1497-1503 (2014).

73. Vitart, V. et al. SLC2A9 is a newly identified urate transporter influencing serum urate concentration, urate excretion and gout. Nat. Genet. 40, 437-442 (2008).

74. Anzai, N. et al. Plasma urate level is directly regulated by a voltage-driven urate efflux transporter URATv1 (SLC2A9) in humans. J. Biol. Chem. 283, 26834-26838 (2008).

75. Caulfield, M. J. et al. SLC2A9 is a high-capacity urate transporter in humans. PLoS Med. 5, e197 (2008).

76. Dinour, D. et al. Homozygous SLC2A9 mutations cause severe renal hypouricemia. J. Am. Soc. Nephrol. 21, 64-72 (2010).

77. Dinour, D. et al. Two novel homozygous SLC2A9 mutations cause renal hypouricemia type 2. Nephrol. Dial. Transplant 27, 1035-1041 (2012).

78. Matsuo, H. et al. Mutations in glucose transporter 9 gene SLC2A9 cause renal hypouricemia. Am. J. Hum. Genet. 83, 795 (2008).

79. Weiss, M. J. et al. A missense mutation in the human liver/bone/kidney alkaline phosphatase gene causing a lethal form of hypophosphatasia. Proc. Natl. Acad. Sci. U. S. A. 85, 7666-7669 (1988). 
80. Sergi, C., Mornet, E., Troeger, J. \& Voigtlaender, T. Perinatal hypophosphatasia: Radiology, pathology and molecular biology studies in a family harboring a splicing mutation $(648+1 \mathrm{~A})$ and a novel missense mutation $(\mathrm{N} 400 \mathrm{~S})$ in the tissue-nonspecific alkaline phosphatase (TNSALP) gene. Am. J. Med. Genet. 103, 235-240 (2001).

81. Wainschtein, P. et al. Recovery of trait heritability from whole genome sequence data. Yearbook of Paediatric Endocrinology (2019) doi:10.1530/ey.16.14.15.

82. Hernandez, R. D. et al. Ultrarare variants drive substantial cis heritability of human gene expression. Nat. Genet. 51, 1349-1355 (2019).

83. Mancuso, N. et al. The contribution of rare variation to prostate cancer heritability. Nat. Genet. 48, 30-35 (2016).

84. Young, A. I. Solving the missing heritability problem. PLoS Genet. 15, e1008222 (2019).

85. Younes, N. et al. A whole-genome sequencing association study of low bone mineral density identifies new susceptibility loci in the phase I Qatar Biobank cohort. J. Pers. Med. 11, 34 (2021).

86. Taliun, D. et al. Sequencing of 53,831 diverse genomes from the NHLBI TOPMed Program. Nature 590, 290-299 (2021).

87. Leitsalu, L. et al. Cohort profile: Estonian Biobank of the Estonian Genome Center, University of Tartu. Int. J. Epidemiol. 44, 1137-1147 (2015).

88. Turro, E. et al. Whole-genome sequencing of patients with rare diseases in a national health system. Nature 583, 96$102(2020)$.

89. Mathieson, I. \& McVean, G. Differential confounding of rare and common variants in spatially structured populations. Nat. Genet. 44, 243-246 (2012).

90. Bhatia, G. et al. Subtle stratification confounds estimates of heritability from rare variants. bioRxiv (2016) doi:10.1101/048181.

91. 1000 Genomes Project Consortium et al. A global reference for human genetic variation. Nature 526, 68-74 (2015).

92. Weissbrod, O. et al. Functionally informed fine-mapping and polygenic localization of complex trait heritability. Nat. Genet. 52, 1355-1363 (2020).

93. Berisa, T. \& Pickrell, J. K. Approximately independent linkage disequilibrium blocks in human populations. Bioinformatics 32, 283-285 (2016).

94. Hou, K. et al. Accurate estimation of SNP-heritability from biobank-scale data irrespective of genetic architecture. Nat. Genet. 51, 1244-1251 (2019). 


\section{Material and Methods}

\section{Model and definitions of estimands}

We model the phenotype of a given individual using a standard linear model, $y=\mathbf{x}^{\mathrm{T}} \boldsymbol{\beta}+\epsilon$, where $\mathbf{x}^{\mathrm{T}}=$ $\left(x_{1} \ldots x_{M}\right)^{\mathrm{T}}$ is the vector of standardized genotypes at $\mathrm{M}$ variants, i.e. $\mathbb{E}\left[x_{i}\right]=0$ and $\operatorname{var}\left[x_{i}\right]=1$ for $i=1, \ldots, M$. $\boldsymbol{\beta}$ is the $\mathrm{M} \times 1$ vector of standardized causal effect sizes, and $\epsilon \sim N\left(0, \sigma_{e}^{2}\right)$ is environmental noise. We assume that the phenotype is standardized in the population, i.e. $\mathbb{E}[y]=0, \operatorname{var}[y]=1$. Linkage disequilibrium (LD) between variants $i$ and $j$ is defined as $r_{i j} \equiv \operatorname{cov}\left[x_{i}, x_{j}\right]=\mathbb{E}\left[x_{i} x_{j}\right]$ and the full LD matrix for all M variants is $\mathbf{R} \equiv$ $\operatorname{cov}\left[\mathbf{x}^{\mathrm{T}}\right]$.

Letting $p_{\text {causal }} \in[0,1]$ such that $M \times p_{\text {causal }}$ is the total number of causal variants, we assume the causal effect of the $i$-th variant is $\beta_{i} \sim N\left(0, \frac{h_{G}^{2}}{M \times p_{\text {causal }}}\right)$ with probability $p_{\text {causal }}$ or $\beta_{i}=0$ with probability $1-p_{\text {causal }}$. Under this model, total SNP-heritability $h_{G}^{2}$ is defined as the proportion of phenotypic variance explained by the M variants,

$$
\begin{aligned}
h_{\mathrm{G}}^{2} & \equiv \frac{\operatorname{var}\left[\mathbf{x}^{\mathrm{T}} \boldsymbol{\beta}\right]}{\operatorname{var}[y]} \\
& =\mathbb{E}_{\boldsymbol{\beta}}\left[\operatorname{var}\left[\mathbf{x}^{\mathrm{T}} \boldsymbol{\beta} \mid \boldsymbol{\beta}\right]\right]+\operatorname{var}_{\boldsymbol{\beta}}\left[\mathbb{E}\left[\mathbf{x}^{\mathrm{T}} \boldsymbol{\beta} \mid \boldsymbol{\beta}\right]\right] \\
& =\mathbb{E}_{\boldsymbol{\beta}}\left[\boldsymbol{\beta}^{\mathrm{T}} \operatorname{var}\left[\mathbf{x}^{\mathrm{T}}\right] \boldsymbol{\beta}\right]+\operatorname{var}_{\boldsymbol{\beta}}\left[\mathbb{E}\left[\mathbf{x}^{\mathrm{T}}\right] \boldsymbol{\beta}\right] \\
& =\mathbb{E}_{\boldsymbol{\beta}}\left[\boldsymbol{\beta}^{\mathrm{T}} \mathbf{R} \boldsymbol{\beta}\right]+\operatorname{var}_{\boldsymbol{\beta}}[0] \\
& =\mathbb{E}_{\boldsymbol{\beta}}\left[\boldsymbol{\beta}^{\mathrm{T}} \mathbf{R} \boldsymbol{\beta}\right]
\end{aligned}
$$

where the second line follows from the Law of Total Variance.

Let $g$ index a gene of interest. Given an assignment of $m_{g}$ variants to gene $g$, let $\mathbf{x}_{g}^{\mathrm{T}}$ be the $m_{g} \times 1$ vector of genotypes at this set of variants and let $\mathbf{x}_{g}^{\mathrm{T}}$, be the genotypes of the remaining $M-m_{g}$ variants. We can rewrite the total SNP-heritability of the trait in terms of gene $g$ as

$$
\begin{aligned}
h_{\mathrm{G}}^{2} & =\operatorname{Var}\left[\mathbf{x}_{g}^{\mathrm{T}} \boldsymbol{\beta}_{g}+\mathbf{x}_{g^{\prime}}^{\mathrm{T}} \boldsymbol{\beta}_{g^{\prime}}\right] \\
& =\operatorname{Var}\left[\mathbf{x}_{g}^{\mathrm{T}} \boldsymbol{\beta}_{g}\right]+\operatorname{Var}\left[\mathbf{x}_{g^{\prime}}^{\mathrm{T}} \boldsymbol{\beta}_{g^{\prime}}\right]+2 \operatorname{Cov}\left[\mathbf{x}_{g}^{\mathrm{T}} \boldsymbol{\beta}_{g}, \mathbf{x}_{g^{\mathrm{T}}}^{\mathrm{T}} \boldsymbol{\beta}_{g^{\prime}}\right] \\
& =\mathrm{E}_{\boldsymbol{\beta}}\left[\boldsymbol{\beta}_{g}^{\mathrm{T}} \mathbf{R}_{g} \boldsymbol{\beta}_{g}\right]+\mathrm{E}_{\boldsymbol{\beta}}\left[\boldsymbol{\beta}_{g^{\prime}}^{\mathrm{T}} \mathbf{R}_{g^{\prime}} \boldsymbol{\beta}_{g^{\prime}}\right]+2\left[\mathrm{E}\left[\left(\mathbf{x}_{g}^{\mathrm{T}} \boldsymbol{\beta}_{g}\right)\left(\mathbf{x}_{g^{\prime}}^{\mathrm{T}} \boldsymbol{\beta}_{g^{\prime}}\right)\right]-\mathrm{E}\left[\mathbf{x}_{g}^{\mathrm{T}} \boldsymbol{\beta}_{g}\right] \mathrm{E}\left[\mathbf{x}_{g^{\prime}}^{\mathrm{T}} \boldsymbol{\beta}_{g^{\prime}}\right]\right] \\
& =\mathrm{E}_{\boldsymbol{\beta}}\left[\boldsymbol{\beta}_{g}^{\mathrm{T}} \mathbf{R}_{g} \boldsymbol{\beta}_{g}\right]+\mathrm{E}_{\boldsymbol{\beta}}\left[\boldsymbol{\beta}_{g^{\prime}}^{\mathrm{T}} \mathbf{R}_{g^{\prime}} \boldsymbol{\beta}_{g^{\prime}}\right]+2 \mathrm{E}_{\boldsymbol{\beta}}\left[\mathrm{E}\left[\left(\mathbf{x}_{g}^{\mathrm{T}} \boldsymbol{\beta}_{g}\right)\left(\boldsymbol{\beta}_{g^{\prime}}^{\mathrm{T}} \mathbf{x}_{g^{\prime}}\right) \mid \boldsymbol{\beta}\right]\right]-2 \mathrm{E}_{\boldsymbol{\beta}}\left[\mathrm{E}\left(\mathbf{x}_{g}^{\mathrm{T}} \boldsymbol{\beta}_{g} \mid \boldsymbol{\beta}\right)\right] \mathrm{E}_{\boldsymbol{\beta}}\left[\mathrm{E}\left(\mathbf{x}_{g^{\prime}}^{\mathrm{T}} \boldsymbol{\beta}_{g^{\prime}} \mid \boldsymbol{\beta}\right)\right] \\
& =\mathrm{E}_{\boldsymbol{\beta}}\left[\boldsymbol{\beta}_{g}^{\mathrm{T}} \mathbf{R}_{g} \boldsymbol{\beta}_{g}\right]+\mathrm{E}_{\boldsymbol{\beta}}\left[\boldsymbol{\beta}_{g^{\prime}}^{\mathrm{T}} \mathbf{R}_{g^{\prime}} \boldsymbol{\beta}_{g^{\prime}}\right]+2 \mathrm{E}_{\boldsymbol{\beta}}\left[\boldsymbol{\beta}_{g} \boldsymbol{\beta}_{g^{\prime}}^{\mathrm{T}} \mathrm{E}\left[\mathbf{x}_{g^{\prime}} \mathbf{x}_{g}^{\mathrm{T}}\right]\right]-0 \\
& =\mathrm{E}_{\boldsymbol{\beta}}\left[\boldsymbol{\beta}_{g}^{\mathrm{T}} \mathbf{R}_{g} \boldsymbol{\beta}_{g}\right]+\mathrm{E}_{\boldsymbol{\beta}}\left[\boldsymbol{\beta}_{g^{\prime}}^{\mathrm{T}} \mathbf{R}_{g^{\prime}} \boldsymbol{\beta}_{g^{\prime}}\right]+2 \mathrm{E}_{\boldsymbol{\beta}}\left[\boldsymbol{\beta}_{g} \boldsymbol{\beta}_{g^{\prime}}^{\mathrm{T}}\right] \mathrm{E}_{\mathbf{x}}\left[\mathbf{x}_{g^{\prime}} \mathbf{x}_{g}^{\mathrm{T}}\right]
\end{aligned}
$$


where the fourth line follows from the Law of Total Expectation. If we additionally assume that $\operatorname{cov}\left[\beta_{i}, \beta_{j}\right]=0$ for all $i \neq j$, then $\mathbb{E}\left[\boldsymbol{\beta}_{(g)} \boldsymbol{\beta}_{\left(g^{\prime}\right)}^{\mathrm{T}}\right]=\operatorname{cov}\left[\boldsymbol{\beta}_{(g)}, \boldsymbol{\beta}_{\left(g^{\prime}\right)}\right]=0$, which simplifies the above equation to

$$
h_{\mathrm{G}}^{2}=\mathbb{E}_{\boldsymbol{\beta}}\left[\boldsymbol{\beta}_{g}^{\mathrm{T}} \mathbf{R}_{g} \boldsymbol{\beta}_{g}\right]+\mathbb{E}_{\boldsymbol{\beta}}\left[\boldsymbol{\beta}_{g^{\prime}}^{\mathrm{T}} \mathbf{R}_{g^{\prime}} \boldsymbol{\beta}_{g^{\prime}}\right]
$$

We refer to the first term, the component of heritability attributable to the causal effects in gene $g$, as total genelevel heritability, i.e.

$$
h_{\text {gene,t }}^{2}=\boldsymbol{\beta}_{g}^{\mathrm{T}} \mathbf{R}_{g} \boldsymbol{\beta}_{g}
$$

Using the same assumptions as above, we can partition the variants in gene $g$ by minor allele frequency such that

$$
h_{\text {gene,t }}^{2}=h_{\text {gene,r }}^{2}+h_{\text {gene,lf }}^{2}+h_{\text {gene,c }}^{2}
$$

where $h_{\text {gene,r }}^{2}, h_{\text {gene,lf }}^{2}$, and $h_{\text {gene,c }}^{2}$ are the components of $h_{\text {gene,t }}^{2}$ attributable to the causal effects of rare (MAF $<$ $0.01)$, low-frequency $(0.01 \leq \mathrm{MAF}<0.05)$, and common $(\mathrm{MAF} \geq 0.05)$ variants, respectively. The estimands of interest in this work are the four terms in $h_{\text {gene,t }}^{2}=h_{\text {gene,r }}^{2}+h_{\text {gene,lf }}^{2}+h_{\text {gene,c }}^{2}$.

\section{Estimating the posterior distribution of gene-level heritability}

Since we have neither the "true" causal effect sizes, $\boldsymbol{\beta}$, nor the population LD, R, we must estimate both from data. We consider one approximately independent LD block at a time. Given a GWAS of $N$ individuals, let $\mathbf{X}=$ $\left[\mathbf{x}_{1}^{\mathrm{T}}, \ldots, \mathbf{x}_{N}^{\mathrm{T}}\right]^{\mathrm{T}}$ be the $N \times M$ matrix of standardized genotypes measured at $M$ variants, let $\mathbf{y}=\left(y_{1}, \ldots, y_{N}\right)^{\mathrm{T}}$ be an $N \times 1$ vector of phenotypes, and let $\boldsymbol{\epsilon} \sim \operatorname{MVN}\left(\mathbf{0}, \sigma_{e}^{2} \mathbf{I}_{N}\right)$ be environmental noise.

It is often the case that individual-level genotype data are inaccessible for privacy or logistical reasons. However, GWAS summary statistics - estimates of the causal effects and their standard errors - are publicly available for thousands of traits. Ordinary least squares (OLS) estimates of the causal effects are often provided, defined as

$$
\widehat{\boldsymbol{\beta}}_{\mathrm{GWAS}}=\frac{1}{N} \mathbf{X}^{\mathrm{T}} \mathbf{y}=\frac{1}{N} \mathbf{X}^{\mathrm{T}}(\mathbf{X} \boldsymbol{\beta}+\boldsymbol{\epsilon})=\frac{1}{N} \mathbf{X}^{\mathrm{T}} \mathbf{X} \boldsymbol{\beta}+\frac{1}{N} \mathbf{X}^{\mathrm{T}} \boldsymbol{\epsilon}
$$

It follows that

$$
p\left(\widehat{\boldsymbol{\beta}}_{\mathrm{GWAS}} \mid \boldsymbol{\beta}, \widehat{\mathbf{R}}, \sigma_{e}^{2}\right) \sim \operatorname{MVN}\left(\widehat{\mathbf{R}} \boldsymbol{\beta}, \frac{\sigma_{e}^{2}}{N} \widehat{\mathbf{R}}\right)
$$

In this scenario, the observed data $\mathrm{D}$ are not the individual-level genotypes and phenotypes $(\mathbf{X}, \mathbf{y})$, but rather $\mathrm{D}=$ $\left(\widehat{\boldsymbol{\beta}}_{\mathrm{GWAS}}, \widehat{\mathbf{R}}\right)$, where $\widehat{\mathbf{R}}$ is an estimate of LD computed from either the genotypes of a set of individuals in the GWAS ("in-sample” LD) or from an external reference panel (e.g., 1000 Genomes ${ }^{91}$ ). By combining the prior on 
$\boldsymbol{\beta}, p(\boldsymbol{\beta} \mid \boldsymbol{\lambda})(\boldsymbol{\lambda}$ represents hyperparameters in the prior over $\boldsymbol{\beta}$, estimated with empirical Bayes procedure as implemented in $\mathrm{SuSiE})$, and the likelihood of the observed data, $p\left(\widehat{\boldsymbol{\beta}}_{\mathrm{GWAS}} \mid \boldsymbol{\beta}, \widehat{\mathbf{R}}, \sigma_{e}^{2}\right)$, one can compute the posterior distribution of the causal effects, $p\left(\boldsymbol{\beta} \mid \widehat{\boldsymbol{\beta}}_{\mathrm{GWAS}}, \widehat{\mathbf{R}}, \boldsymbol{\lambda}, \sigma_{e}^{2}\right)$. The hyperparameters $\boldsymbol{\lambda}$ and $\sigma_{e}^{2}$ can be estimated with an empirical Bayes procedure as in $\mathrm{SuSiE}$ framework. We note that for computational efficiency, we can partition the whole genome into approximately independent LD blocks, and estimate the posterior distribution of $\boldsymbol{\beta}$ separately for each LD block. Because each LD block is approximately independent of the rest of the genomes by definition, the genetic effects from SNPs outside of the LD block of interest are absorbed into the environmental noise. And correspondingly, the LD block-specific hyperparameters $\left(\lambda, \sigma_{e}^{2}\right)$ are estimated independently for each LD block.

The posterior of $\boldsymbol{\beta}, p(\boldsymbol{\beta} \mid \mathrm{D})$, is in general computationally intractable. Approximate inference, e.g., Markov Chain Monte Carlo (MCMC) or variance inference, can be used to approximate the exact posterior $p(\boldsymbol{\beta} \mid \mathrm{D})$ as $\tilde{p}(\boldsymbol{\beta} \mid \mathrm{D})$. In this work, we use $\mathrm{SuSiE}^{47}$, a variational inference-based implementation of linear regression with sparse prior. (In principle, it is straightforward to use other implementations of linear regression with sparse prior). We draw $K$ samples from the posterior of the causal effects, $\widetilde{\boldsymbol{\beta}}^{(1)}, \ldots, \widetilde{\boldsymbol{\beta}}^{(K)} \sim \tilde{p}(\boldsymbol{\beta} \mid \mathrm{D})$. This approximate distribution can in turn be used to approximate the full posterior distribution of $h_{\text {gene }}^{2}$, i.e. $\left(\widetilde{\boldsymbol{\beta}}_{g}^{(1)}\right)^{\mathrm{T}} \widehat{\mathbf{R}}_{g}\left(\widetilde{\boldsymbol{\beta}}_{g}^{(1)}\right), \ldots,\left(\widetilde{\boldsymbol{\beta}}_{g}^{(K)}\right)^{\mathrm{T}} \widehat{\mathbf{R}}_{g}\left(\widetilde{\boldsymbol{\beta}}_{g}^{(K)}\right)$. Finally, given the approximate posterior of $h_{\text {gene }}^{2}$, one can compute the posterior mean,

$$
\begin{aligned}
& \hat{h}_{\text {gene }}^{2}=\widehat{\mathrm{E}}\left[\boldsymbol{\beta}_{g}^{\mathrm{T}} \mathbf{R}_{g} \boldsymbol{\beta}_{g} \mid \mathrm{D}\right] \\
& \approx \frac{1}{K} \sum_{k=1}^{K}\left(\widetilde{\boldsymbol{\beta}}_{g}^{(k)}\right)^{\mathrm{T}} \widehat{\mathbf{R}}_{g}\left(\widetilde{\boldsymbol{\beta}}_{g}^{(k)}\right)
\end{aligned}
$$

and measures of uncertainty such as credible intervals (described below). Similar procedures could be applied to estimate the gene-level heritabilities stratified by annotations of SNPs (such as MAF-based annotation).

\section{Quantifying uncertainty in gene-level heritability estimates}

$\widetilde{\boldsymbol{\beta}}^{(1)}, \ldots, \widetilde{\boldsymbol{\beta}}^{(K)}$ provide an approximation to the full posterior distribution of $\boldsymbol{\beta}$, thus capturing uncertainty about the causal effect sizes arising from two main sources: LD and finite GWAS sample size (Figure 1). Therefore, by using the full posterior of $\boldsymbol{\beta}$ to approximate the full posterior of $h_{\mathrm{gene}}^{2}$, we wish to capture uncertainty in the causal effects that propagates into our estimate of $h_{\text {gene }}^{2}$. (The noise in $\widehat{\mathbf{R}}$ is also an important factor but, for simplicity, we first investigate uncertainty in $\hat{h}_{\text {gene }}^{2}$ in simulations where $\widehat{\mathbf{R}}=\mathbf{R}$.)

We summarize the uncertainty in $h_{\text {gene }}^{2}$ by computing $\rho$-level credible intervals ( $\rho$-CIs). For a given $\rho \in[0,1], \rho$ CI is defined as the central interval within which $h_{\text {gene }}^{2}$ lies with probability $\rho$, i.e. the upper and lower bounds of 
$\rho$-CI are set to the empirical $\frac{1-\rho}{2}$ and $1-\left(\frac{1-\rho}{2}\right)$ quantiles of the posterior samples $\left(\widetilde{\boldsymbol{\beta}}_{g}^{(k)}\right)^{\mathrm{T}} \widehat{\mathbf{R}}_{g}\left(\widetilde{\boldsymbol{\beta}}_{g}^{(k)}\right), k=$ $1, \ldots, K$.

\section{Implementation details}

We partition the genome into approximately independent LD blocks ${ }^{93}$ and, for each gene of interest, we perform inference on the LD block containing the gene. For each LD block, we extract the marginal association statistics and estimate LD for all the variants in the LD block. We estimate the posterior distribution of effect sizes using the function "susie_suff_stat" with default parameters, as implemented in $\mathrm{SuSiE}^{47}$ v0.8. We use the function “susie_get_posterior_samples” to obtain 500 posterior samples.

\section{Simulation framework}

We obtain the real imputed genotypes of $\mathrm{N}=290,273$ "unrelated white British" individuals in the UK Biobank by extracting individuals with self-reported British ancestry who are $>$ third-degree relatives (pairs of individuals with kinship coefficient $<1 / 2^{(9 / 2)}$, as defined in ref. ${ }^{48}$ ). Filtering on MAF $>0.5 \%$ leaves 200,235 variants on chromosome 1. A list of 1,083 genes on chromosome 1 and their coordinates were downloaded from https://github.com/bogdanlab/gene_sets (Data Availability). For each variant, genotypes are standardized such that the mean is 0 and variance is 1 across individuals. Phenotypes were simulated under a variety of genetic architectures according to the following steps. First, we randomly select $3 \%, 8 \%$, or $16 \%$ (out of the 1,083 genes) to be causal $\left(h_{\text {gene }}^{2}>0\right)$. Second, we draw causal variants in the causal gene bodies and within 10-kb upstream/downstream of the gene start/end positions; the causal variants in the window around the gene are intended to represent regulatory causal variants in transcription start sites (TSSs). The causal configuration is set to be either (1) 5 causal variants in gene body and 3 causal variants in TSS or (2) 10 causal variants in gene body and 6 causal variants in TSS. Third, we draw noncoding "background" causal variants across the whole chromosome with frequency $p_{\text {causal }}=\{0.001,0.01\}$. Finally, conditional on the causal statuses of the variants, we draw independent causal effect sizes from a Gaussian distribution where the variance of each causal variant is standardized such that the gene bodies collectively have a heritability of 3\%, TSSs collectively have 1\%, and noncoding background variants together explain $1 \%$. We note that the causal statuses and effect sizes for each variant are only drawn once; the environmental noise term is drawn 30 times independently to generate 30 simulation replicates.

\section{Evaluating and comparing gene-level heritability estimates in simulations}

Recall that for a given gene $g$, the causal effect sizes and LD of the variants assigned to the gene are denoted $\boldsymbol{\beta}_{g}$ and $\mathbf{R}_{g}$, and ground-truth gene-level heritability is defined as $h_{\text {gene }}^{2}=\boldsymbol{\beta}_{g}^{\mathrm{T}} \mathbf{R}_{g} \boldsymbol{\beta}_{g}$. The posterior mean estimated for a single simulation replicate $s$ is denoted $\hat{h}_{\text {gene,(s) }}^{2}$. We estimate the bias of the estimator as bias $\left[\hat{h}_{\text {gene }}^{2}\right] \approx$ 
$\frac{1}{30} \sum_{s}\left(\hat{h}_{\text {gene,(s) }}^{2}-h_{\text {gene }}^{2}\right)$; the variance of the estimator as $\operatorname{Var}\left[\hat{h}_{\text {gene }}^{2}\right] \approx \frac{1}{30} \sum_{s}\left(\hat{h}_{\text {gene,(s) }}^{2}-h_{\text {gene }}^{2}\right)^{2}$; and the mean squared error as MSE $\left[\hat{h}_{\text {gene }}^{2}\right]=\left(\operatorname{bias}\left[\hat{h}_{\text {gene }}^{2}\right]\right)^{2}+\operatorname{Var}\left[\hat{h}_{\text {gene }}^{2}\right]$.

For each simulation replicate $s$, we also output $\rho$-level credible intervals, defined as $\mathrm{CI}_{(s)}=\left(\hat{h}_{\text {gene }, \frac{1-\rho}{2},(\mathrm{~s})}{ }^{2}\right.$ $\left.\hat{h}_{\text {gene,1- } \frac{1-\rho}{2},(\mathrm{~s})}^{2}\right)$, where the $\frac{1-\rho}{2}$ and $1-\left(\frac{1-\rho}{2}\right)$ quantiles are estimated from the posterior samples. To assess the accuracy of credible intervals, we calculate empirical coverage across simulation replicates, defined as the proportion of simulation replicates in which the $\rho$-level credible interval covers the ground-truth gene-level heritability: $\frac{1}{30} \sum_{s} \mathbb{I}\left[\hat{h}_{\text {gene,(s) }}^{2} \in \mathrm{CI}_{(s)}\right]$.

\section{Comparison to "naïve" gene-level heritability estimator}

We compare our approach to an alternative "naïve" estimator of gene-level heritability that does not model LD between the gene and its adjacent regions and thus ignores causal-effect uncertainty. This estimator is similar to existing methods that are meant to be applied to approximately independent LD blocks ${ }^{45,94}$. For each gene $g$, we extract the marginal association statistics, $\widehat{\boldsymbol{\beta}}_{g}$, and the estimated LD, $\widehat{\mathbf{R}}_{g}$, for the variants assigned to the gene, and we compute the alternative estimator as $\frac{N \widehat{\boldsymbol{\beta}}_{g}^{\top} \widehat{\mathbf{R}}_{g}^{\dagger} \widehat{\boldsymbol{\beta}}_{g}^{\top}}{N-q}$, where $\widehat{\mathbf{R}}_{g}^{\dagger}$ and $q$ are the pseudo-inverse and rank of $\widehat{\mathbf{R}}_{g}$, respectively ${ }^{45,94}$.

\section{Assessing robustness to $L D$ panel sample size}

To assess the robustness of our approach to the sample size of the LD panel used to estimate LD, we randomly draw a subset of $\mathrm{N}=\{500,1000,2500,5000\}$ individuals from the full 290,273 individuals. After extracting variants with MAF $>0.5 \%$, genotypes are standardized to have mean 0 and variance 1 , similar to the full-sample analysis. Since we are interested in assessing robustness to noisy estimates of LD, all analyses are performed using the same set of marginal association statistics used in the full-sample analysis, excluding the variants that were filtered from the LD panel based on MAF. The LD and marginal association statistics are fed into the h2gene software, similar to the full-sample analysis.

\section{Analysis of 25 UK Biobank phenotypes}

We analyzed 25 quantitative phenotypes in the UK Biobank. Phenotypes and imputed genotypes were filtered according to the same procedures used in the simulation analyses, leaving $\mathrm{N}=290,273$ individuals and $\mathrm{M}=5,650,812$ variants with $\mathrm{MAF}>0.5 \%$. Quantitative phenotypes were quantile-normalized to a Gaussian distribution with mean 0 and variance 1 . We then performed a GWAS for each trait using the "assoc" option in PLINK (Web Resources) with age, sex, and the top 10 genetic principal components included as covariates. We computed in-sample LD for each approximately independent LD block ${ }^{93}$. We downloaded gene names and 
coordinates from https://github.com/bogdanlab/gene sets and, for each gene, we define the estimand of interest to be a function of the variants in the gene body and those located within 10-kb upstream/downstream of the gene start/end positions. Finally, given the in-sample LD and marginal association statistics, we infer the posterior distribution of the causal effect sizes one LD block at a time, and we estimate and partition gene-level heritability for all genes in each LD block, where we define the estimand of interest to be a function of the variants in the gene body and those located within 10-kb upstream/downstream of the gene start/end positions. MAGMA v1.09 was used for gene-level association with a $10 \mathrm{~kb}$ window around each gene. The same list of genes and the same set of imputed variants were used for the MAGMA analysis. 


\section{Figures and Tables}

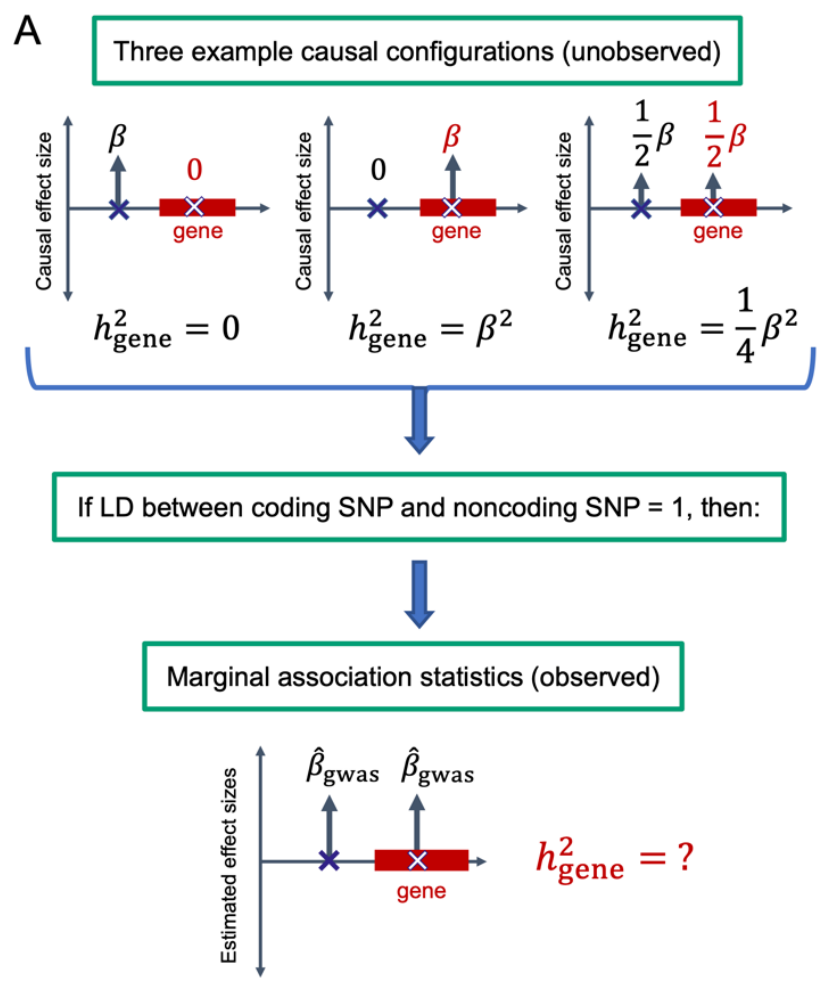

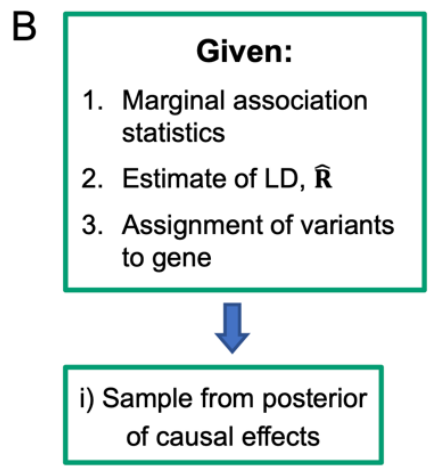
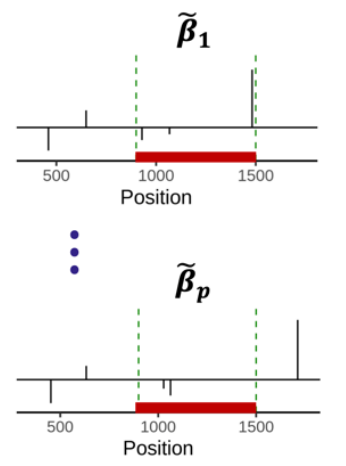
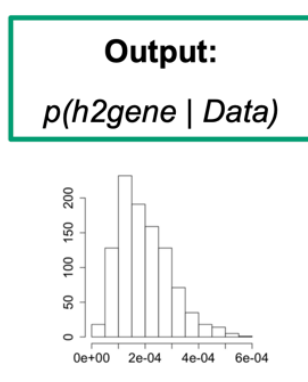

$\tilde{h}_{\text {gene(1) }}^{2}=\widetilde{\boldsymbol{\beta}}_{1}^{\mathrm{T}} \mathbf{R} \widetilde{\boldsymbol{\beta}}_{1}$

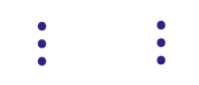

$\tilde{h}_{\text {gene }(p)}^{2}=\widetilde{\boldsymbol{\beta}}_{p}^{\mathrm{T}} \mathbf{R} \widetilde{\boldsymbol{\beta}}_{p}$

ii) Estimate h2gene for each posterior sample

Figure 1. Overview. (A) Toy example with two variants, one of which is assigned to the gene of interest. The top row depicts 3 example causal configurations corresponding to 3 different gene-level heritabilities $\left(0, \beta^{2}\right.$, and $\left.\beta^{2} / 4\right)$. Since the variants in are in perfect $L D$, all 3 causal configurations yield the same expected marginal association statistics. (B) Given marginal association statistics, an estimate of LD, and an assignment of variants to the gene of interest, our approach involves i) sampling from the posterior of the causal effect sizes (assuming a sparse prior) to capture our uncertainty about which variants are causal, and then ii) estimating gene-level heritability for each posterior sample to approximate the posterior distribution of gene-level heritability. 

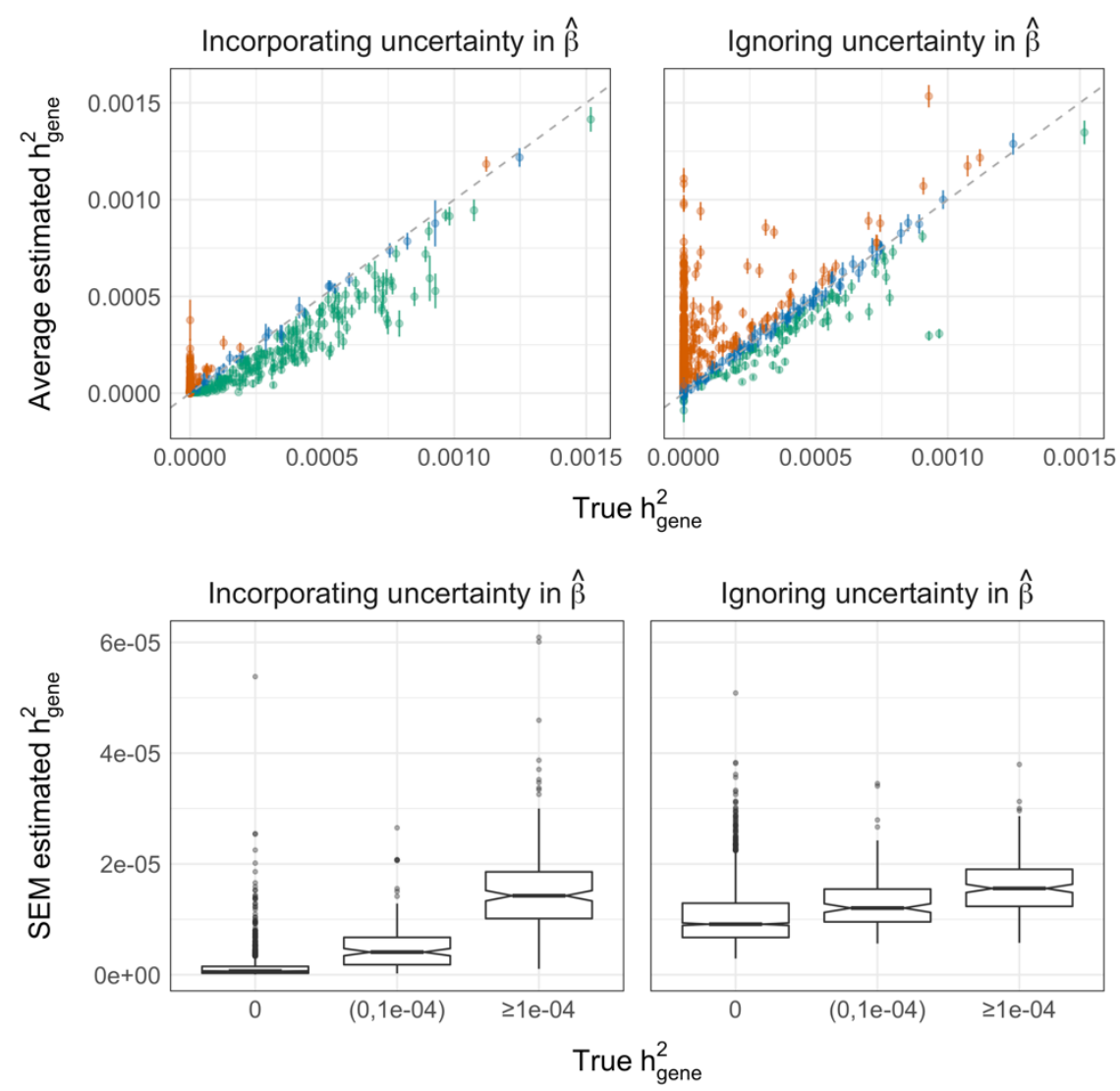

Figure 2. Impact of uncertainty in the estimated causal effects on gene-level heritability estimation in simulations. Chromosome $1, \mathrm{MAF}>0.5 \%, p_{\text {causal }}=0.01, \mathrm{~N}=290 \mathrm{~K}$ individuals, and 1,038 genes, of which $16 \%$ have nonzero gene-level heritability. Top row: each point is the average $\hat{h}_{\text {gene }}^{2}$ for a given gene across 30 simulation replicates; error bars mark $1.96 \times$ standard error of the mean (SEM). Orange and green points are genes for which the estimator is significantly upward-biased and downward-biased, respectively. Bottom row: distributions of SEM with respect to gene-level heritability. 


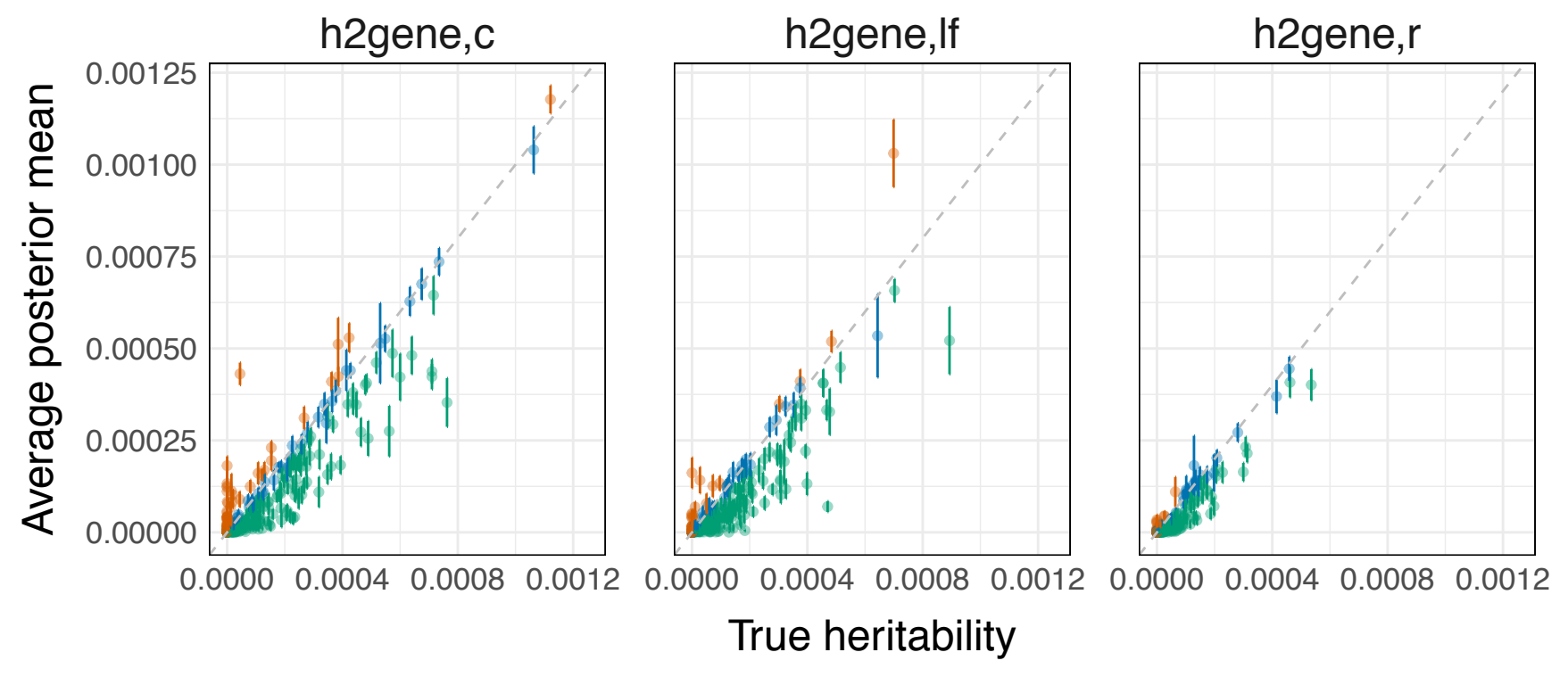

Figure 3. Estimates of the heritability contributions of common, low-frequency, and rare variants in simulations. Chromosome $1, \mathrm{MAF}>0.5 \%, p_{\text {causal }}=0.01, \mathrm{~N}=290 \mathrm{~K}$ individuals, and 1,083 genes, of which $16 \%$ have nonzero heritability. Each point is the average posterior mean for a given gene from 30 simulation replicates; error bars mark 1.96 x SEM. Orange and green points are genes for which the estimator is significantly upward-biased and downward-biased, respectively, where significance is determined by the error bars. 


\begin{tabular}{|c|c|c|c|c|c|c|}
\hline Trait & $h_{\text {gene }, t}^{2}>0$ & $\geq \frac{1}{2} \sum h_{\text {gene }, t}^{2}$ & $(\%)$ & $\begin{array}{l}h_{\text {gene }, t}^{2} \\
=h_{\text {gene,c }}^{2}\end{array}$ & $\begin{array}{l}h_{\text {gene }, t}^{2} \\
=h_{\text {gene,lf }}^{2}\end{array}$ & $\begin{array}{l}h_{\text {gene }, t}^{2} \\
=h_{\text {gene, } r}^{2}\end{array}$ \\
\hline Corneal Hysteresis & 1212 & 42 & $3.5 \%$ & 912 & 82 & 4 \\
\hline Hair Color & 1328 & 6 & $0.5 \%$ & 972 & 92 & 14 \\
\hline BMD Heel T-score & 1430 & 48 & $3.4 \%$ & 1098 & 90 & 4 \\
\hline Alkaline Phosphatase & 1695 & 9 & $0.5 \%$ & 1257 & 120 & 20 \\
\hline SHBG & 1699 & 5 & $0.3 \%$ & 1277 & 118 & 19 \\
\hline $\mathrm{MCH}$ & 1701 & 41 & $2.4 \%$ & 1253 & 137 & 18 \\
\hline C-reactive Protein & 1702 & 5 & $0.3 \%$ & 1293 & 98 & 7 \\
\hline apoA-I & 1730 & 14 & $0.8 \%$ & 1290 & 119 & 14 \\
\hline Platelet Distribution Width & 1736 & 19 & $1.1 \%$ & 1316 & 117 & 20 \\
\hline MSCV & 1738 & 38 & $2.2 \%$ & 1339 & 118 & 11 \\
\hline Urate & 1744 & 2 & $0.1 \%$ & 1319 & 119 & 14 \\
\hline Monocyte Count & 1750 & 41 & $2.3 \%$ & 1332 & 112 & 10 \\
\hline HDL & 1766 & 14 & $0.8 \%$ & 1321 & 126 & 11 \\
\hline GGT & 1784 & 37 & $2.1 \%$ & 1361 & 108 & 13 \\
\hline $\mathrm{HbA1c}$ & 1813 & 26 & $1.4 \%$ & 1345 & 145 & 17 \\
\hline $\begin{array}{l}\text { High Light Scatter } \\
\text { Reticulocyte Count }\end{array}$ & 1858 & 56 & $3.0 \%$ & 1399 & 129 & 25 \\
\hline IGF1 & 1859 & 62 & $3.3 \%$ & 1402 & 128 & 12 \\
\hline Body Mass Index (BMI) & 1879 & 184 & $9.8 \%$ & 1430 & 116 & 8 \\
\hline Cystatin C & 1900 & 22 & $1.2 \%$ & 1452 & 121 & 9 \\
\hline Platelet Count & 1910 & 64 & $3.4 \%$ & 1471 & 119 & 25 \\
\hline Forced Vital Capacity & 1910 & 157 & $8.2 \%$ & 1465 & 123 & 6 \\
\hline Mean Platelet Volume & 1912 & 32 & $1.7 \%$ & 1408 & 140 & 25 \\
\hline RBC Count & 1915 & 89 & $4.6 \%$ & 1461 & 138 & 21 \\
\hline Basal Metabolic Rate & 2099 & 181 & $8.6 \%$ & 1608 & 128 & 11 \\
\hline Height & 2469 & 168 & $6.8 \%$ & 1860 & 182 & 32 \\
\hline
\end{tabular}

Table 1. Summary of nonzero-heritability genes $(90 \%-\mathrm{Cl})$ for 25 quantitative traits. Columns 1-4: complex trait; total number of nonzero-heritability genes (out of 17,437 ), defined as having (i) $h_{\text {gene,t }}^{2}$ $90 \%-\mathrm{Cl}>0$ and (ii) $90 \%-\mathrm{Cl}>0$ for at least one MAF bin (rare, low-frequency, or common); number (and \%) of nonzero-heritability genes that explain at least $50 \%$ of cumulative $h_{\text {gene,t }}^{2}$ for the trait. Columns 5-7: numbers of genes with nonzero heritability contributions exclusively from common, lowfrequency, or rare variants. (BMD = bone mineral density; $\mathrm{MCH}=$ mean corpuscular hemoglobin; $\mathrm{MSCV}=$ mean sphered corpuscular volume; $\mathrm{RBC}=$ red blood cell.) 


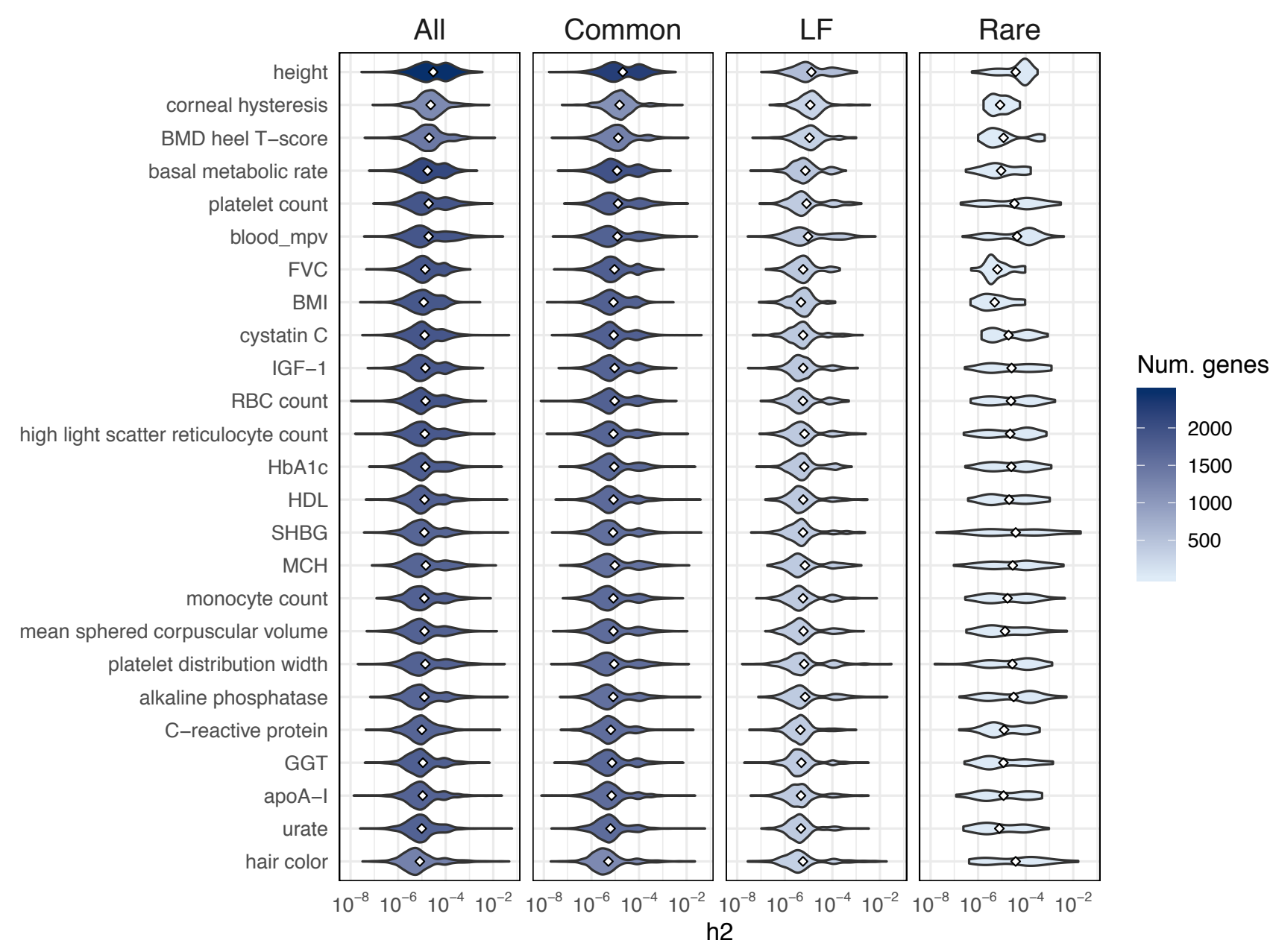

Figure 4. Distributions of h2 estimates for 25 traits. Each violin plot is the distribution of posterior mean estimates for genes with $90 \%-\mathrm{Cl}>0$ for one trait. The shading scales with the number of genes in the violin plot. 


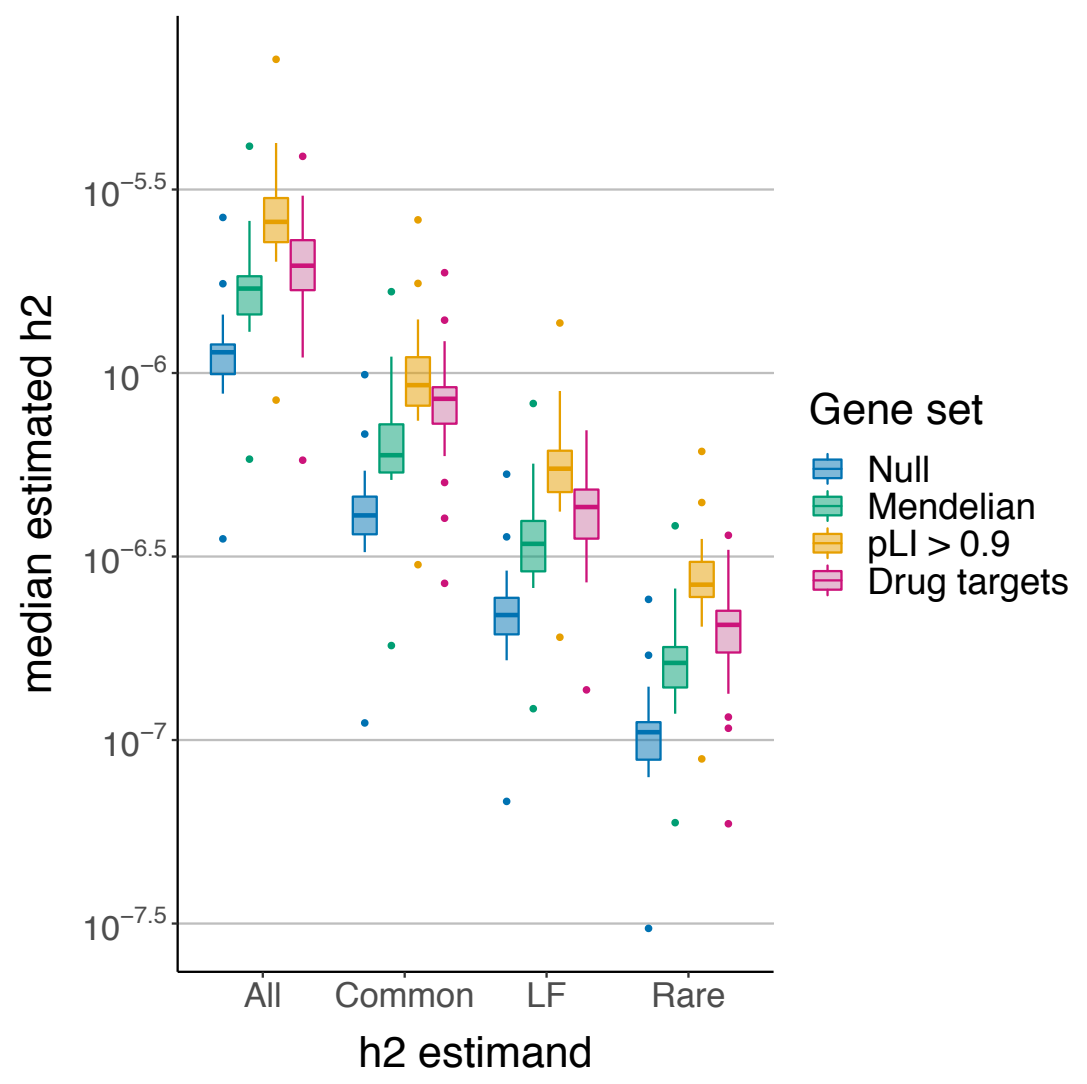

Figure 5. Distributions of h2 estimates for 3 gene sets. Mendelian-disorder genes $(n=3,446)$, LoFintolerant genes $(n=3,230)$, and immune-related drug targets $(n=216)$. Each point is the median posterior mean across genes for a given trait; each boxplot contains 25 quantitative traits in the UK Biobank. 

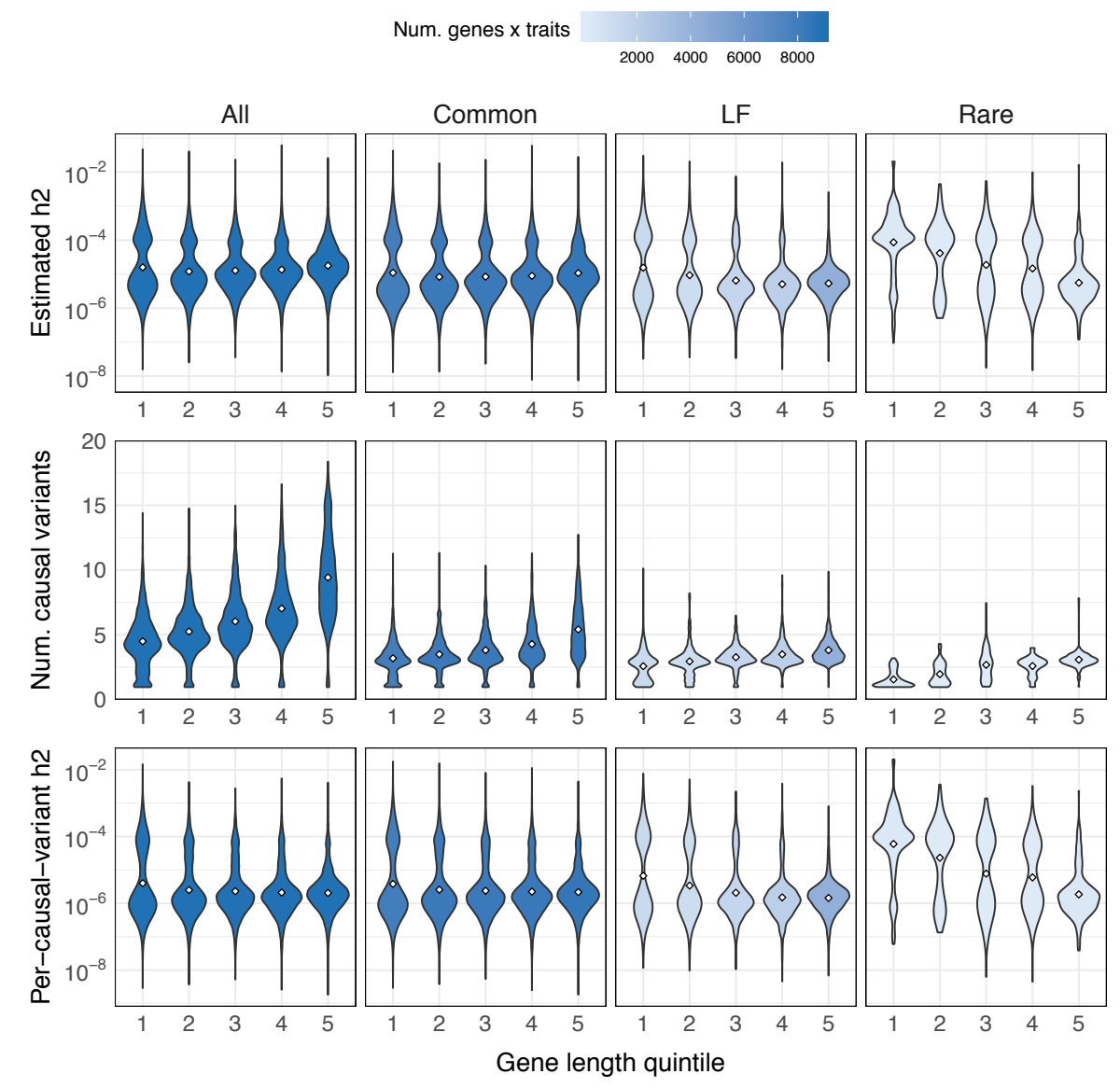

Figure 6. Inverse relationship between rare-variant h2 estimates and gene length. Estimates of h2 (top), number of causal variants per gene (middle), and expected effect size per causal variant per gene (bottom) with respect to gene length (x-axis) for 25 traits. Each violin plot is the distribution of posterior mean estimates for nonzero-heritability genes with $90 \%-C l s>0$ for each h2 quantity. Color gradient indicates the number of estimates in each violin plot (number of gene-trait pairs). 
a

$$
\begin{array}{ll}
\text { - urate - alkaline phosphatase }- \text { HbA1c } \\
\text { - GGT - HDL }
\end{array}
$$

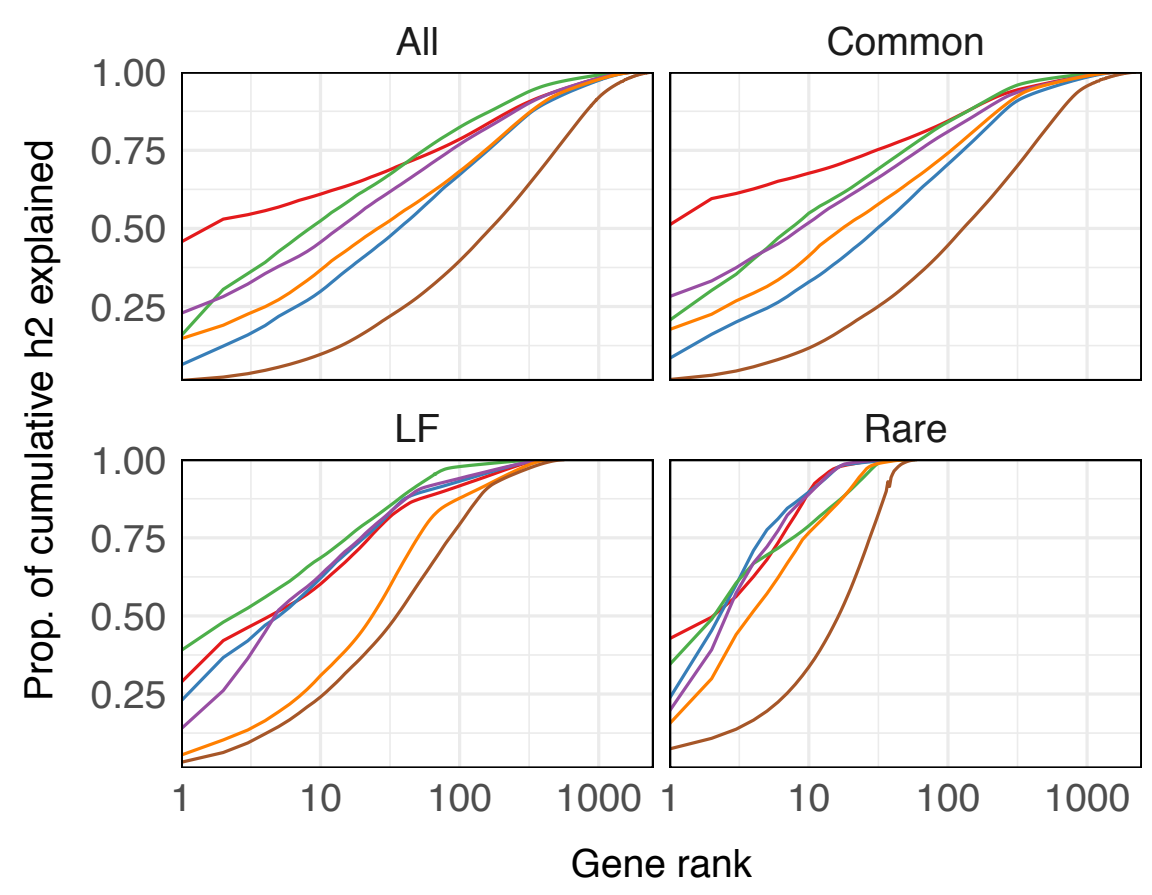

b Prop. causal genes with h2 > expected

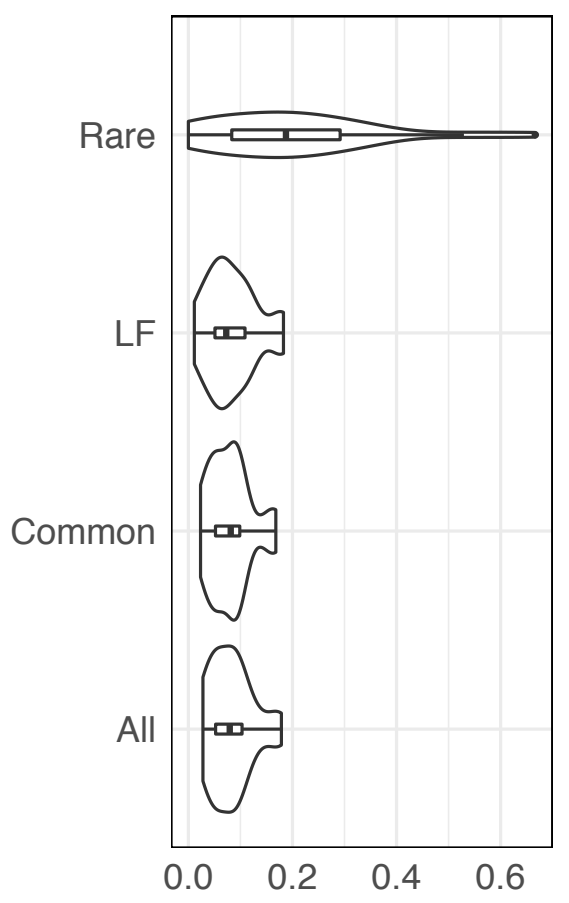

Figure 7. Gene-level heritability estimates capture differences polygenicity across traits. (a) Empirical distributions of cumulative heritability for six example traits (clockwise from top left: total, common, low-frequency, and rare). Each curve can be read as, "the top $\mathrm{X}$ genes explain $\mathrm{Y} \%$ of the cumulative gene-level heritability for a given trait." Cumulative gene-level h2 is estimated by summing the estimated posterior means for nonzero-h2 genes $(90 \%-\mathrm{Cl}>0)$. (Supplementary Figure 21 shows all 25 traits.) (b) Proportion of nonzero-h2 genes per trait with disproportionately large heritability estimates, defined as genes with $90 \%-\mathrm{Cl}>$ (cumulative heritability / number of causal genes)). Each violin plot represents 25 traits. 